\title{
Spectra of glueballs and oddballs and the equation of state from holographic QCD
}

\author{
Lin Zhang $\odot,{ }^{1, *}$ Chutian Chen $\oplus^{2, \dagger}$ Yidian Chen $\odot,{ }^{1, *}$ and Mei Huang $\oplus^{1, \S}$ \\ ${ }^{1}$ School of Nuclear Science and Technology, University of Chinese Academy of Sciences, \\ Beijing, People's Republic of China 100049 \\ ${ }^{2}$ School of Physical Sciences, University of Chinese Academy of Sciences, \\ Beijing, People's Republic of China 100049
}

(Received 21 October 2021; accepted 3 January 2022; published 18 January 2022)

\begin{abstract}
We study the spectra of two-gluon glueballs and three-gluon oddballs and corresponding equation of state in 5-dimensional deformed holographic QCD models in the gravity-dilaton system, where the metric, the dilaton field, and the dilaton potential are self-consistently solved from each other through the Einstein field equations and the equation of motion of the dilaton field. We compare the models by inputting the dilaton field, inputting the deformed metric, and inputting the dilaton potential, and find that with only 2 parameters, the 5-dimensional holographic QCD model predictions on glueballs/oddballs spectra, in general, are in good agreement with lattice results except three oddballs states $0^{+-}, 2^{+-}$and $3^{--}$. From the results of glueballs/oddballs spectra at zero temperature and the equation of state at finite temperature, we observe that the model with quadratic dilaton field can simultaneously describe glueballs/oddballs spectra as well as the equation of state of pure gluon system. The model with quadratic $A_{E}(z)$ can describe glueballs/oddballs spectra, but its corresponding equation of state behaves more like $N_{f}=2+1$ quark matter, which is consistent with the dimension analysis at ultraviolet (UV) boundary. Our results suggest that the Einstein-Maxwell-dilaton model with the profile $\phi(z)=z^{2}$ can be regarded as a candidate of dual theory of pure gluodynamics. Though it is still difficult to find the dual theory of full QCD, the existence of dual theory of pure gluodynamics would be quite encouraging.
\end{abstract}

DOI: 10.1103/PhysRevD.105.026020

\section{INTRODUCTION}

Glueball is one of the most crucial predictions from quantum chromodynamics (QCD), whose non-Abelian feature makes it possible to form bound states of gauge bosons, i.e., glueballs made of two/three gluons (gg, ggg, etc.), [1]. The gauge field plays a more important dynamical role in glueballs than that in the standard hadrons, therefore studying particles like glueballs offers a good opportunity of understanding nonperturbative aspects of QCD. The glueball spectra has attracted much attention for four decades [1], and it has been widely investigated by using various nonperturbative methods. For example, glueballs have been studied by using lattice QCD [2-11], by using effective models like flux

\footnotetext{
*zhanglin@ucas.ac.cn

chenchutian18@mails.ucas.ac.cn

chenyidian@ucas.ac.cn

§huangmei@ucas.ac.cn
}

Published by the American Physical Society under the terms of the Creative Commons Attribution 4.0 International license. Further distribution of this work must maintain attribution to the author(s) and the published article's title, journal citation, and DOI. Funded by SCOAP . tube model [12] and MIT bag model [13-17], by using QCD sum rules [18-32] as well as by using the relativistic manybody approach [33-35]. There are also some other analyses of glueballs in Refs. [36-44]. For more information, please refer to review papers [45-47].

On the other hand, the spin and mass of the glueball can be constrained from high energy scattering data. Regge trajectories $\alpha(t)=\alpha_{0}+\alpha^{\prime} t$ of the glueball have been used to fit high energy pp and $p \bar{p}$ scattering cross section. The $C$-parity even glueball, Pomeron exchange gives the lightest $J=2^{++}$glueball mass $M=\sqrt{t}=1.92 \mathrm{GeV}$. Analogy with the "Pomeron," $C$-parity odd "odderon" contributing to large odd amplitude was proposed in the 1970s in describing the high energy $p p$ and $p \bar{p}$ scattering $[48,49]$. The odderon was regarded as a three-gluon state:

$$
O_{a b c}^{\mu \nu \sigma}(k 1, k 2, k 3)=d_{a b c} G_{a}^{\mu}\left(k_{1}\right) G_{b}^{\nu}(k 2) G_{c}^{\nu}(k 3)
$$

where the lower indices refer to color and the upper ones refer to the Lorentz structure, and $d_{a b c}$ is the fundamental symmetric tensor in $\mathrm{SU}(3)$. The evidence for the identification of the odderon has been debated for a longtime. Recently, the D0 collaboration and TOTEM Collaboration announced the evidence of a $t$-channel exchanged $C$-parity 
odd odderons in $p p$ and $p \bar{p}$ scattering [50]. Especially the odderon's contribution at the dip-bump region is very essential. The mass of $3^{--}$odderon $M_{3^{--}}=3.001 \mathrm{GeV}$ and dacay width $\Gamma_{3^{--}}=2.984 \mathrm{GeV}$ are extracted by using the dipole (DP) Regge model to fit the scattering data [51-54].

In Ref. [32], the oddball spectra has been calculated by using the QCD sum rule. In this work, we are going to investigate the glueball spectra in the framework of holographic QCD, which is based on the gravity/gauge duality, or anti-de Sitter/conformal field theory (AdS/CFT) correspondence [55-57]. AdS/CFT correspondence offers a new possibility to tackle the difficulty of strongly coupled gauge theories [58-61]. Many efforts from both top-down and bottom-up approaches have been paid on examining the nonperturbative properties of QCD [62], e.g., QCD equation of state [63-65], phase transitions [66-72], fluid properties of quark-gluon plasma, meson spectra [72-84], baryon spectra [85-87], as well as the glueball sector [88-111]. In Refs. [112,113], by linearizing the fluctuations around a classical $\sigma$-model coupled to gravity in $d+1$ dimensions, a gauge invariant (diffeomorphism invariant) formalism for calculating the spectra of scalar glueballs and tensor glueballs was developed, which was initially proposed in Refs. [114-116]. This algorithmic formalism was tested and some nontrivial applications were given in Refs. [117-126]. The glueball mass spectra and decay rate in the Sakai-Sugimoto model have been investigated in Refs. [127-129]. Glueballs and oddballs spectra have also been widely studied by using the bottom-up approach, where some studies are based on hard-wall [73] and softwall holographic QCD models [74] with the conformal $\mathrm{AdS}_{5}$ background metric.

A realistic nonconformal holographic QCD model should reveal both the spontaneous chiral symmetry breaking and color charge confinement or linear confinement, which are two main features of QCD in the low energy regime. In the top-down approach, the Sakai-Sugimoto (SS) model or $D_{4}-D_{8}$ brane system $[75,76]$ is one of the most successful nonconformal holographic QCD models. In the bottom-up approach, the dynamical holographic QCD (DhQCD) model constructed in Refs. [67,102,130] can simultaneously describe both chiral symmetry breaking and linear confinement, where the gluon dynamics background is solved by the coupling between the gravity and the dilaton field $\Phi(z)$, which is responsible for the gluon condensate and confinement, and the scalar field $X(z)$ is introduced to mimic chiral dynamics. Evolution of the dilaton field and scalar field in 5-dimensional space-time resemble the renormalization group from ultraviolet (UV) to infrared (IR). This dynamical holographic QCD model describes the scalar glueball spectra and the light meson spectral quite well $[67,102,130]$. Except the dynamical holographic QCD model, there are several other nonconformal holographic QCD models in the same gravity-dilaton system which can well describe nonperturbative QCD properties, e.g., the Gubser model [131-133] and the improved holographic QCD model [134-136] with inputting of a dilaton potential, and the refined model [137] and Dudal model [138] with inputting of a deformed metric.

In the gravity-dilaton system, the metric, the dilaton field, and the dilaton potential are self-consistently solved from each other through the Einstein field equations and the equation of motion of the dilaton field. In principle, the three types of models, (A) inputting the form of the dilaton field, (B) inputting the deformed metric, and (C) inputting the dilaton potential, should be equivalent to describe the background at zero temperature and zero density. We will compare the spectra of glueballs/oddballs including scalar, vector, as well as tensor states and their excitations with those from the lattice QCD, and compare thermodynamic properties with the lattice QCD results for pure gluon system and/or $2+1$ flavors system in these three types of models. Though these models have been separately investigated on thermodynamics and scalar/tensor glueballs, however, it is still worthy to check the consistency of the models and whether they can simultaneously describe the glueball spectra and the equation of state. Only those models which can simultaneously describe the glueball spectra and pure gluon system's equation of state are candidates of dual theory of gluodynamics. It is still difficult to find the dual theory of full QCD, the existence of dual theory of pure gluodynamics would be quite encouraging.

The paper is organized as follows: we introduce the general Einstein-Maxwell-dilaton framework in Sec. II. Then in Sec. III we introduce five different models in the gravity-dilaton system. In Sec. IV we introduce the glueball and oddball operator and calculate the mass spectra in these models and we compare the results of mass spectra with lattice results, results from the QCD sum rule, and results extracted from high energy scattering data. In Sec. V we compare thermodynamic properties of these models with lattice results. Finally, a summary is given in Sec. VI.

\section{THE GENERAL EINSTEIN-MAXWELL- DILATON SYSTEM}

To keep the self-consistency of investigating the glueball spectra as well as further studies on QCD matter at finite temperature and finite chemical potential, we first introduce the general framework of the Einstein-Maxwell-dilaton (EMD) system, which comes back to the gravity-dilaton coupling system at zero chemical potential. The total action of the 5-dimensional holographic QCD model including glueball/oddball excitations is

$$
S_{\text {total }}^{s}=S_{b}^{s}+S_{g}^{s}
$$

where $S_{b}^{s}$ is the action for the background in the string frame, and $S_{g}^{s}$ is the action describing glueballs in the string frame. 
The Einstein-Maxwell-dilaton action $S_{b}^{s}$ for the background in the string frame is

$$
\begin{aligned}
S_{b}^{s}= & \frac{1}{2 \kappa_{5}^{2}} \int \mathrm{d}^{5} x \sqrt{-g^{s}} e^{-2 \Phi}\left[R^{s}+4 g^{s M N} \partial_{M} \Phi \partial_{N} \Phi\right. \\
& \left.-V^{s}(\Phi)-\frac{h(\Phi)}{4} e^{\frac{4 \Phi}{3}} g^{s M \tilde{M}} g^{s N \tilde{N}} F_{M N} F_{\tilde{M} \tilde{N}}\right],
\end{aligned}
$$

where $s$ denotes the string frame, $\kappa_{5}^{2}=8 \pi G_{5}$, the $G_{5}$ is the 5-dimensional Newton constant. The $g^{s}$ is the determinant of the metric in the string frame: $g^{s}=\operatorname{det}\left(g_{M N}\right)$, and the metric tensor in the string frame is extracted from

$$
d s^{2}=\frac{L^{2} e^{2 A_{s}(z)}}{z^{2}}\left(-f(z) d t^{2}+\frac{d z^{2}}{f(z)}+d y_{1}^{2}+d y_{3}^{2}+d y_{3}^{2}\right),
$$

where $L$ is the curvature radius of the asymptotic $\mathrm{AdS}_{5}$ space-time. For simplicity, we set $L=1$ in the following calculations. The $R^{s}$ is the Ricci curvature scalar in the string frame. The scalar field $\Phi(z)$ is the dilaton field which depends only on the coordinate $z, F_{M N}$ is the field strength of the $U(1)$ gauge field $A_{M}$ :

$$
F_{M N}=\partial_{M} A_{N}-\partial_{N} A_{M}
$$

The 5-dimensional field $A_{M}$ is dual to baryon number current. $h(\Phi)$ describes the coupling strength of $A_{M}$ in the theory, $V^{s}(\Phi)$ represents the potential of the dilaton field in the string frame. $h(\Phi)$ and $V^{s}(\Phi)$ are the functions that depends only on the value of $\Phi$.

\section{A. The Einstein-Maxwell-dialton system in the Einstein frame}

As discussed in Ref. [139], it is convenient to calculate the vacuum expectation value of the loop operator in the string frame, and it is more convenient to work out the gravity solution and to study equation of state in the Einstein frame. So we apply the Weyl transformation $[140,141]$

$$
g_{M N}^{S}=\mathrm{e}^{\frac{4}{3} \Phi} g_{M N}^{E}
$$

to Eq. (3). Here $g_{M N}^{E}$ is the metric tensor in the Einstein frame, the capital letter "E" denotes the Einstein frame. Then, Eq. (3) can be written as

$$
\begin{aligned}
S^{E}= & \frac{1}{2 \kappa_{5}^{2}} \int \mathrm{d}^{5} x \sqrt{-g^{E}}\left[R^{E}-\frac{4}{3} g^{E M N} \partial_{M} \Phi \partial_{N} \Phi-V^{E}(\Phi)\right. \\
& \left.-\frac{h(\Phi)}{4} g^{E M \tilde{M}} g^{E N \tilde{N}} F_{M N} F_{\tilde{M} \tilde{N}}\right]
\end{aligned}
$$

with $V^{E}=\mathrm{e}^{\frac{4}{3} \Phi} V^{s}$.
Then we define a new dilaton field $\phi$ :

$$
\phi=\sqrt{\frac{8}{3}} \Phi
$$

Now Eq. (7) becomes

$$
\begin{aligned}
S^{E}= & \frac{1}{2 \kappa_{5}^{2}} \int \mathrm{d}^{5} x \sqrt{-g^{E}}\left[R^{E}-\frac{1}{2} g^{E M N}\left(\partial_{M} \phi\right)\left(\partial_{N} \phi\right)-V_{\phi}(\phi)\right. \\
& \left.-\frac{h_{\phi}(\phi)}{4} g^{E M \tilde{M}} g^{E N \tilde{N}} F_{M N} F_{\tilde{M} \tilde{N}}\right]
\end{aligned}
$$

where $V_{\phi}(\phi)=V^{E}(\Phi)$, and $h_{\phi}(\phi)=h(\Phi)$. According to Eqs. (4), (6), and (8), we can derive the line element in Einstein frame:

$d s^{2}=\frac{L^{2} e^{2 A_{E}(z)}}{z^{2}}\left(-f(z) d t^{2}+\frac{d z^{2}}{f(z)}+d y_{1}^{2}+d y_{3}^{2}+d y_{3}^{2}\right)$,

where

$$
A_{E}(z)=A_{s}(z)-\sqrt{\frac{1}{6}} \phi(z) .
$$

After applying variation to Eq. (9), we can derive the Einstein field equations and the equations of motion of $A_{M}$ and $\phi$ as follows

$$
\begin{aligned}
R_{M N}^{E}-\frac{1}{2} g_{M N}^{E} R^{E}-T_{M N} & =0, \\
\nabla_{M}\left[h_{\phi}(\phi) F^{M N}\right] & =0,
\end{aligned}
$$$$
\partial_{M}\left[\sqrt{-g} \partial^{M} \phi\right]-\sqrt{-g}\left(\frac{\mathrm{d} V_{\phi}(\phi)}{\mathrm{d} \phi}+\frac{F^{2}}{4} \frac{\mathrm{d} h_{\phi}(\phi)}{\mathrm{d} \phi}\right)=0,
$$

with the energy-momentum tensor $T_{M N}$

$$
\begin{aligned}
T_{M N}= & \frac{1}{2}\left[\left(\partial_{M} \phi\right)\left(\partial_{N} \phi\right)-\frac{1}{2} g_{M N}^{E} g^{E P \tilde{P}}\left(\partial_{P} \phi\right)\left(\partial_{\tilde{P}} \phi\right)\right. \\
& \left.-g_{M N}^{E} V_{\phi}(\phi)\right]+\frac{h_{\phi}(\phi)}{2}\left(g^{E P \tilde{P}} F_{M P} F_{N \tilde{P}}\right. \\
& \left.-\frac{1}{4} g_{M N}^{E} g^{E P \tilde{P}} g^{E Q \tilde{Q}} F_{P Q} F_{\tilde{P} \tilde{Q}}\right) .
\end{aligned}
$$

We can safely suppose all the components of $A_{M}(z)$ are zero except $A_{t}(z)$. Substituting Eq. (10) into the EOMs Eq. (12), we then derive the EOMs for the components:

$$
\begin{array}{r}
A_{t}^{\prime \prime}+A_{t}^{\prime}\left(-\frac{1}{z}+\frac{h_{\phi}{ }^{\prime}}{h_{\phi}}+A_{E}{ }^{\prime}\right)=0, \\
f^{\prime \prime}+f^{\prime}\left(-\frac{3}{z}+3 A_{E}^{\prime}\right)-\frac{e^{-2 A_{E}} A_{t}^{\prime 2} z^{2} h_{\phi}}{L^{2}}=0,
\end{array}
$$




$$
\begin{gathered}
A_{E}^{\prime \prime}+\frac{f^{\prime \prime}}{6 f}+A_{E}^{\prime}\left(-\frac{6}{z}+\frac{3 f^{\prime}}{2 f}\right)-\frac{1}{z}\left(-\frac{4}{z}+\frac{3 f^{\prime}}{2 f}\right) \\
+3 A_{E}^{\prime 2}+\frac{L^{2} e^{2 A_{E}} V_{\phi}}{3 z^{2} f}=0, \\
A_{E}^{\prime \prime}-A_{E}^{\prime}\left(-\frac{2}{z}+A_{E}^{\prime}\right)+\frac{\phi^{\prime 2}}{6}=0, \\
\phi^{\prime \prime}+\phi^{\prime}\left(-\frac{3}{z}+\frac{f^{\prime}}{f}+3 A_{E}^{\prime}\right)-\frac{L^{2} e^{2 A_{E}}}{z^{2} f} \frac{\mathrm{d} V_{\phi}(\phi)}{\mathrm{d} \phi} \\
+\frac{z^{2} e^{-2 A_{E}} A_{t}^{\prime 2}}{2 L^{2} f} \frac{\mathrm{d} h_{\phi}(\phi)}{\mathrm{d} \phi}=0 .
\end{gathered}
$$

In the above five equations, only four of them are independent. Thus we can choose Eq. (18) as a constraint, which can be used to check the solutions.

\section{FIVE DIFFERENT MODELS IN THE EMD SYSTEM}

In the gravity-dilaton system, the metric, the dilaton field, and the dilaton potential can be self-consistently solved from each other through the Einstein field equations and the equation of motion of the dilaton field. At zero temperature and zero chemical potential, the function $f(z)=1$ and $A_{t}(z)=0$, then Eq. (14) to Eq. (18) can be simplified:

$$
\begin{array}{r}
A_{E}^{\prime \prime}-\frac{6}{z} A_{E}^{\prime}+\frac{4}{z^{2}}+3 A_{E}^{2}+\frac{L^{2} e^{2 A_{E}} V_{\phi}}{3 z^{2}}=0, \\
A_{E}^{\prime \prime}-A_{E}^{\prime}\left(-\frac{2}{z}+A_{E}^{\prime}\right)+\frac{\phi^{\prime 2}}{6}=0, \\
\phi^{\prime \prime}+\phi^{\prime}\left(-\frac{3}{z}+3 A_{E}^{\prime}\right)-\frac{L^{2} e^{2 A_{E}}}{z^{2}} \frac{\mathrm{d} V_{\phi}(\phi)}{\mathrm{d} \phi}=0,
\end{array}
$$

where Eq. (21) is the constraint. Under the condition that we have proper boundary conditions, if we input (A) the form of the dilaton field $\phi(z)$, or (B) the function $A_{E}(z)$, or (C) the dilaton potential $V_{\phi}(\phi)$, we can solve the other two. In principle, these three types of models of EMD system are totally equivalent to describe the background in the vacuum. However, at finite temperature and finite chemical potential, the situation will become different. If we input $V_{\phi}(\phi)$, the form of $V_{\phi}(\phi)$ is independent of the temperature/chemical potential, from Eq. (14) and Eq. (18), we can solve different functions $A_{E}(z)$ and $\phi(z)$ at different temperature/chemical potential, which can be denoted by $A_{E T, \mu}(T, \mu, z)$ and $\phi_{T, \mu}(T, \mu, z)$. On the other hand, if we input $A_{E}(z)$ [or $\phi(z)$ ], whose form is independent of temperature/chemical potential, we can derive $V_{\phi}(\phi)$ with temperature/chemical potential dependence, which can be denoted by $V_{\phi T, \mu}(T, \mu, \phi)$. The two descriptions, that are equivalent at vacuum, now become distinct from each other at finite temperature/chemical potential. From now on, we call fixing $V_{\phi}(\phi)$ "description A," fixing $A_{E}(z)$ or $\phi(z)$ is denoted by "description B."

It is more convenient to solve the system in the Einstein frame from Eqs. (19)-(21). In the following we list two sets of vacuum solutions of $V_{\phi}(\phi), A_{E}(z)$, and $\phi(z)$ that satisfy the EOMs.

\section{A. Vacuum solutions: Set $\mathcal{A}$}

From the experiences in Refs. [137,138], we can input the function $A_{E}(z)$ in the Einstein frame, and solve $V_{\phi}(\phi)$ and $\phi(z)$. The simplest ansatz for the deformed metric is $A_{E}(z)=-a z^{2}$, and from Eqs. (19)-(21) one can derive the solution as following:

$$
\begin{aligned}
A_{E}(z) & =-a z^{2}, \\
V_{\phi}(\phi) & =-\frac{6}{L^{2}} \mathrm{e}^{2(k(\phi))^{2}}\left(6(k(\phi))^{4}+5(k(\phi))^{2}+2\right), \\
\phi(z) & =z \sqrt{3 a\left(3+2 a z^{2}\right)}+\frac{3}{2} \sqrt{6} \operatorname{arcsinh}\left[\sqrt{\frac{2 a}{3} z}\right],
\end{aligned}
$$

where the auxiliary function $k(\varphi)$ is defined as the inverse function of

$$
\varphi(\mathfrak{z})=\mathfrak{z} \sqrt{3\left(3+2 \mathfrak{z}^{2}\right)}+\frac{3}{2} \sqrt{6} \operatorname{arcsinh}\left[\sqrt{\frac{2}{3}} \mathfrak{z}\right],
$$

which means $k(\varphi(z))=z$ with $z=\sqrt{a} z$. Starting from any of the above three functions, together with proper boundary conditions, we can solve other two functions from Eq. (19) and Eq. (20).

From Eq. (24) we know that $\phi(z=0)=0$, $\lim _{z \rightarrow+\infty} \phi(z) \rightarrow+\infty$. At UV boundary $(z \rightarrow 0)$, the asymptotic forms of $V_{\phi}(\phi)$ and $\phi$ are given below:

$$
\begin{aligned}
L^{2} V_{\phi}(\phi \rightarrow 0)= & -12-\frac{3}{2} \phi^{2}-\frac{1}{12} \phi^{4}-\frac{377}{174960} \phi^{6} \\
& -\frac{977}{33067440} \phi^{8}-\frac{53483}{214277011200} \phi^{10} \\
& -\frac{1564351}{1145524901875200} \phi^{12}+\mathcal{O}\left(\phi^{14}\right),
\end{aligned}
$$

$$
\begin{aligned}
\phi(z \rightarrow 0)= & 6 \sqrt{a} z+\frac{2}{3} a^{\frac{3}{2}} z^{3}-\frac{1}{15} a^{\frac{5}{2}} z^{5}+\frac{1}{63} a^{\frac{7}{2}} z^{7} \\
& -\frac{5}{972} a^{\frac{9}{2}} z^{9}+\frac{7}{3564} a^{\frac{11}{2}} z^{11}-\frac{7}{8424} a^{\frac{13}{2}} z^{13} \\
& +\mathcal{O}\left(z^{15}\right) .
\end{aligned}
$$


From the UV asymptotic form of $V_{\phi}(\phi)$, we can extract the 5-dimensional mass square of $\phi$

$$
M_{\phi}^{2}=-3 \text {. }
$$

According to the mass-dimension relationship $M^{2}=$ $(\Delta-q)(\Delta+q-4)$ and $q=0$, the dimension

$$
\Delta_{\phi_{-}}=1, \quad \Delta_{\phi_{+}}=3 .
$$

At IR boundary $(z \rightarrow+\infty), V_{\phi}(\phi)$ and $\phi(z)$ behave as

$$
\begin{aligned}
L^{2} V_{\phi}(\phi \rightarrow+\infty) & =-\frac{27}{4}\left(\frac{3}{8}\right)^{\frac{1}{4}} \mathrm{e}^{-\frac{3}{2}} \mathrm{e}^{\frac{\sqrt{6}}{3} \phi+\cdots}\left(\phi^{\frac{1}{2}}+\cdots\right), \\
\phi(z \rightarrow+\infty)= & \sqrt{6}\left[a z^{2}+\frac{3}{4}\left(1+\ln \left(\frac{8}{3}\right)+\ln \left(a z^{2}\right)\right)\right. \\
& \left.+\frac{9}{32} \frac{1}{a z^{2}}+\mathcal{O}\left(\frac{1}{z^{4}}\right)\right] .
\end{aligned}
$$

Equation (23) lead to the masses of glueballs $m_{n}$ behave as

$$
m_{n} \sim n^{\frac{1}{2}}, \quad \text { when } n \rightarrow+\infty \text {. }
$$

which shows the linear Regge behavior along $n$.

\section{B. Vacuum solutions: Set $\mathcal{B}$}

As for another set of solution, we start from the form of $\phi(z)$. One simple but nontrivial ansatz is to take the quadratic form of $\phi(z): \phi(z)=b z^{2}$. As discussed in Refs. $[67,102,130,139]$, the quadratic form of the dilaton field is dual to a dimension-2 gluon condensation operator, which is responsible for the linear confinement of the gluon system. Then the solution $V_{\phi}(\phi), A_{E}(z)$ and $\phi(z)$ take the form of

$$
\begin{gathered}
\phi(z)=b z^{2}, \\
V_{\phi}(\phi)=\frac{1}{L^{2}} 2 \times 2^{\frac{3}{4}} \times 3^{\frac{1}{4}} \phi^{\frac{3}{2}}\left[\Gamma\left(\frac{5}{4}\right)\right]^{2}\left\{\left[I_{\frac{1}{4}}\left(\frac{\phi}{\sqrt{6}}\right)\right]^{2}\right. \\
\left.-4\left[I_{-\frac{3}{4}}\left(\frac{\phi}{\sqrt{6}}\right)\right]^{2}\right\}, \\
A_{E}(z)=-\ln \left[\frac{2^{\frac{3}{8}} \times 3^{\frac{1}{8}} \Gamma\left(\frac{5}{4}\right) I_{\frac{1}{4}}\left(\frac{b z^{2}}{\sqrt{6}}\right)}{b^{\frac{1}{4}} \sqrt{z}}\right],
\end{gathered}
$$

where $\Gamma(z)$ is the Euler gamma function, $I_{n}(z)$ is the modified Bessel function of the first kind.

From Eq. (33) we know that $\phi(z=0)=0$, $\lim _{z \rightarrow+\infty} \phi(z) \rightarrow+\infty$. At UV boundary $(z \rightarrow 0)$, the asymptotic forms are

$$
\begin{aligned}
L^{2} V_{\phi}(\phi \rightarrow 0)= & -12-2 \phi^{2}-\frac{4}{15} \phi^{4}-\frac{49}{6075} \phi^{6}-\frac{11}{94770} \phi^{8} \\
& -\frac{11}{11153700} \phi^{10}-\frac{38}{6851160225} \phi^{12} \\
& +\mathcal{O}\left(\phi^{14}\right), \\
A_{E}(z \rightarrow 0)= & -\frac{1}{30} b^{2} z^{4}+\frac{1}{4050} b^{4} z^{8}-\frac{4}{1184625} b^{6} z^{12} \\
+ & \mathcal{O}\left(z^{16}\right) .
\end{aligned}
$$

From the UV asymptotic form of $V_{\phi}(\phi)$, we can extract the 5-dimensional mass square of $\phi$

$$
M_{\phi}^{2}=-4
$$

According to the mass-dimension relationship $M^{2}=$ $(\Delta-q)(\Delta+q-4)$ and $q=0$, the dimension

$$
\Delta_{\phi_{-}}=\Delta_{\phi_{+}}=2 .
$$

At IR boundary $(z \rightarrow+\infty), V_{\phi}(\phi)$ and $A_{E}(z)$ behave as

$$
\begin{aligned}
L^{2} V_{\phi}(\phi \rightarrow \infty)= & -\frac{1}{\pi} 2^{\frac{5}{4}} \times 3^{\frac{7}{4}}\left[\Gamma\left(\frac{5}{4}\right)\right]^{2} \mathrm{e}^{\frac{\sqrt{6}}{3} \phi} \phi^{\frac{1}{2}} \\
& \times\left[1-\frac{23 \sqrt{6}}{48} \frac{1}{\phi}+\mathcal{O}\left(\frac{1}{\phi^{2}}\right)\right] \\
& \times\left\{1+\frac{5 \sqrt{2}}{6} \mathrm{e}^{-\frac{\sqrt{6}}{3} \phi}\left[1+\mathcal{O}\left(\frac{1}{\phi}\right)\right]\right\}, \\
A_{E}(z \rightarrow \infty)=- & \frac{\sqrt{6}}{6} b z^{2}+\frac{3}{2} \ln (\sqrt{b} z)+\ln \left[\frac{\pi^{\frac{1}{2}}}{2^{\frac{1}{8}} 3^{\frac{3}{3}} \Gamma\left(\frac{5}{4}\right)}\right] \\
& -\frac{3 \sqrt{6}}{32} \frac{1}{b z^{2}}+\mathcal{O}\left(\frac{1}{z^{4}}\right)+\mathcal{O}\left(\mathrm{e}^{-\frac{\sqrt{6}}{3} b z^{2}}\right) \\
+ & \mathcal{O}\left(\frac{1}{z^{2}} \mathrm{e}^{-\frac{\sqrt{6}}{3} b z^{2}}\right) .
\end{aligned}
$$

Again, from the asymptotic expansion of $V_{\phi}(\phi)$ at IR boundary, we can conclude that linear Regge behavior of the masses of glueballs $m_{n}$ :

$$
m_{n}^{2} \sim n, \quad \text { when } n \rightarrow+\infty,
$$

which shows the linear Regge behavior along $n$.

\section{Five different models}

The two sets of vacuum solutions listed above have linear confinement and can produce glueball bound state. Not all models can show such feature. According to Refs. [136,142], if we require that the theory is confined and bad singularities are absent, the asymptotic behavior of $V_{\phi}(\phi)$ at IR boundary should be 


$$
\begin{gathered}
L^{2} V_{\phi}(\phi \rightarrow+\infty)=c_{V} \mathrm{e}^{c_{\phi, 1} \phi+\cdots}\left(\phi^{c_{\phi, 2}}+\cdots\right), \\
\times \begin{cases}\frac{\sqrt{6}}{3}<c_{\phi, 1}<\frac{2 \sqrt{3}}{3}, & c_{\phi, 2} \text { is real number }, \\
c_{\phi, 1}=\frac{\sqrt{6}}{3}, & c_{\phi, 2} \geqslant 0,\end{cases}
\end{gathered}
$$

where $c_{V}$ is constant. When $c_{\phi, 1}=\frac{\sqrt{6}}{3}$ and $c_{\phi, 2}>0$, the behavior of the glueball spectra is

$$
m_{n} \sim n^{c_{\phi, 2}} \quad \text { when } n \rightarrow+\infty
$$

If $c_{\phi, 2}=\frac{1}{2}$, it becomes asymptotically linear Regge behavior.

For comparison, we plot three different dilaton potentials $V_{\phi}(\phi)$ in Fig. 1. One of them is the Gubser model taken from Ref. [133]:

$$
V_{\phi}(\phi)=\frac{-12 \cosh (0.606 \phi)+2.057 \phi^{2}}{L^{2}}
$$

the others two are Eq. (23) and Eq. (34). Here we set $L=1$. The dashed black line is $\mathrm{e}^{\frac{\sqrt{6}}{3}} \phi$. According to the conclusion in Sec. III A, if the potential is more gradual than this line, such as the blue line that represents the Gubser model in Eq. (45), the theory is gapless and nonconfining.

As we stated below Eqs. (19)-(21), there are two different descriptions of the input of EMD system. Combining with the two different sets of vacuum solutions Eqs. (23)-(24) and Eqs. (34)-(33), we consider five models in this article.

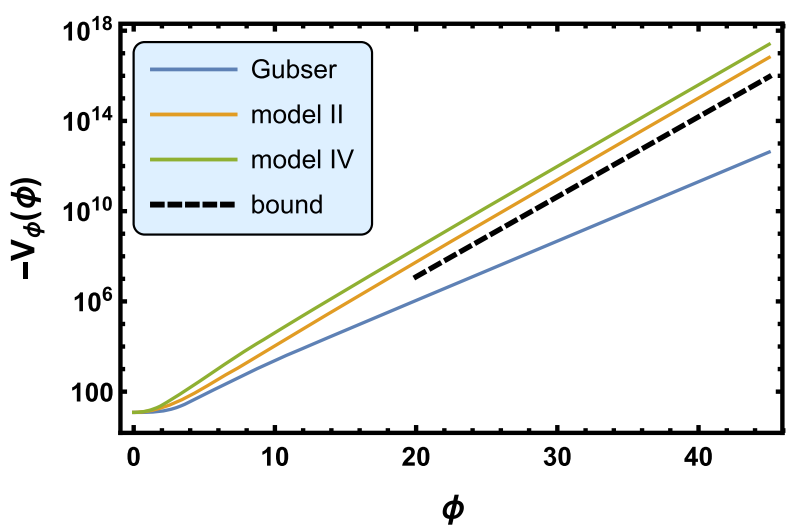

FIG. 1. These are three different dilaton potentials $V_{\phi}(\phi)$. The longitudinal axis is the value of $-V_{\phi}(\phi)$ in logarithm coordinate. The horizontal axis is the value of $\phi$. The dashed black line is $\mathrm{e}^{\frac{\sqrt{6}}{3} \phi}$. The blue line, orange line, and green line represent the potential in Eq. (45), Eq. (23), and Eq. (34) respectively. The meaning of "model II" and "model IV" will be explained later. The dashed black line is $\mathrm{e}^{\frac{\sqrt{6}}{3} \phi}$. The bound is given by Eq. (44) from Refs. [136,142]. If the potential is more gradual than this bound, the theory is gapless and nonconfining.

\section{Model I and II}

In model I, we use description-B and input $A_{E}(z)$ as Eq. (22):

$$
A_{E}(z)=-a z^{2}
$$

Note that the dimension of the parameter $a$ is $[E]^{2}$ and its value decides the energy scale of the EMD system. According to Ref. [138], the simple quadratic form of $A_{E}(z)$ can produce Hawking/Page phase transition, which is dual to confinement/deconfinement phase transition. At vacuum, we use the boundary condition

$$
\phi(z=0)=0 .
$$

Combining the boundary condition Eq. (47) with the EOMs Eq. (20) and Eq. (19), we can solve $\phi(z)$ and $V_{\phi}(\phi)$. The results are Eq. (24) and Eq. (23).

As for finite temperature and finite chemical potential, the EOMs are Eq. (14)-(17). There may exist the black hole [143] in space-time manifold, the metric of which in conformal coordinate $z$ is Eq. (10). The boundary conditions are given as

$$
\begin{aligned}
A_{t}(z=0) & =\mu, \\
A_{t}\left(z=z_{h}\right) & =0, \\
f(z=0) & =1, \\
f\left(z=z_{h}\right) & =0, \\
\phi(z=0) & =0,
\end{aligned}
$$

where $z=z_{h}$ is the location of the event horizon of black hole on the coordinate $z, \mu$ is the chemical potential. Besides the boundary condition Eq. (48), the form of $h_{\phi}(\phi)$ are also needed to solve the EOMs. However, we consider the $\mu=0$ case, which means $A_{t}(z)=0$ through the article. Thus our calculations and results are independent on $h_{\phi}(\phi)$.

In model II, we use description-A and input $V_{\phi}(\phi)$ as Eq. (23):

$V_{\phi}(\phi)=-\frac{6}{L^{2}} \mathrm{e}^{2(k(\phi))^{2}}\left(6(k(\phi))^{4}+5(k(\phi))^{2}+2\right)$.

At vacuum, we use the boundary conditions

$$
\begin{gathered}
A_{E}(z=0)=0, \\
\phi(z=0)=0, \\
\left.\frac{\mathrm{d} \phi(z)}{\mathrm{d} z}\right|_{z=0}=6 \sqrt{a} .
\end{gathered}
$$

Equation (50) guarantees the space-time is asymptotic $\mathrm{AdS}_{5}$ at UV boundary. Equation (52) contains a parameter $a$, the 
dimension of which is $[E]^{2}$ and the value of which decides the energy scale of the EMD system. Given these boundary conditions and the value of $a$ in Eq. (52) being same with that in Eq. (46), we can solve the EOMs at vacuum, then it will be found that the solutions are totally equivalent to those in model I at vacuum.

As for finite temperature and finite chemical potential, the EOMs are Eq. (14)-(17). The boundary conditions are given as

$$
\begin{aligned}
A_{t}(z=0) & =\mu, \\
A_{t}\left(z=z_{h}\right) & =0, \\
f(z=0) & =1, \\
f\left(z=z_{h}\right) & =0, \\
A_{E}(z=0) & =0, \\
\phi(z=0) & =0, \\
\left.\frac{\mathrm{d} \phi(z)}{\mathrm{d} z}\right|_{z=0} & =6 \sqrt{a},
\end{aligned}
$$

where $z=z_{h}$ is the location of the event horizon of black hole on the coordinate $z, \mu$ is the chemical potential. We should emphasize here that at finite temperature or finite chemical potential case, the solutions here are different from those in model I.

\section{Model III and IV}

In model III, we use description B and input $\phi(z)$ as Eq. (33):

$$
\phi(z)=b z^{2} .
$$

Note that the dimension of the parameter $b$ is $[E]^{2}$ and its value decides the energy scale of the EMD system. According to Ref. [74], the desired linear confinement $m_{n, S}^{2} \sim(n+S)$ of mesons at large radially excited quantum number $n$ or large orbitally excited quantum number $S$ can be reproduced in the background metric, in which the large $z$ (IR) asymptotic expansion of $\Phi$ is $\Phi \sim z^{2}$. At the same time, according to Ref. [109], the quadratic dilaton $\Phi(z)=z^{2}$ in the IR can also leads to confinement and an approximate linear glueball spectrum. Substituting Eq. (54) into Eq. (20), we can solve a general solution for $A_{E}(z)$ with two integration constants. However, the value of this general solution is usually complex. If we force the reality of $A_{E}(z)$ and consider the boundary condition

$$
A_{E}(z=0)=0,
$$

we can derive $A_{E}(z)$ and $V_{\phi}(\phi)$. The results are Eq. (35) and Eq. (33).

As for finite temperature and finite chemical potential, the EOMs are Eqs. (14)-(17). The boundary conditions are imposed as

$$
\begin{aligned}
A_{t}(z=0) & =\mu, \\
A_{t}\left(z=z_{h}\right) & =0, \\
f(z=0) & =1, \\
f\left(z=z_{h}\right) & =0,
\end{aligned}
$$

where $z=z_{h}$ is the location of the event horizon of black hole on the coordinate $z, \mu$ is the chemical potential. Collecting these boundary conditions and Eq. (54), Eq. (35), we can then solve the EMD system.

In model IV, we use description A and input $V_{\phi}(\phi)$ as Eq. (34):

$$
\begin{aligned}
V_{\phi}(\phi)= & \frac{1}{L^{2}} 2 \times 2^{\frac{3}{4}} \times 3^{\frac{1}{4}} \phi^{\frac{3}{2}}\left[\Gamma\left(\frac{5}{4}\right)\right]^{2}\left\{\left[I_{\frac{1}{4}}\left(\frac{\phi}{\sqrt{6}}\right)\right]^{2}\right. \\
& \left.-4\left[I_{-\frac{3}{4}}\left(\frac{\phi}{\sqrt{6}}\right)\right]^{2}\right\} .
\end{aligned}
$$

At vacuum, we use the boundary conditions

$$
\begin{gathered}
A_{E}(z=0)=0, \\
\lim _{z \rightarrow 0} \frac{\phi(z)}{z^{2}}=b,
\end{gathered}
$$

Again, we force that $A_{E}(z)$ is real. The Eq. (58) guarantees the space-time is asymptotic $\mathrm{AdS}_{5}$ at UV boundary. Equation (59) contains a parameter $b$, the dimension of which is $[E]^{2}$ and the value of which decides the energy scale of the EMD system. Given these boundary conditions and the value of $b$ in Eq. (59) being same with that in Eq. (54), we can solve the EOMs at vacuum, then it will be found that the solutions are totally equivalent to those in model III at vacuum.

As for finite temperature and finite chemical potential, the EOMs are Eqs. (14)-(17). The boundary conditions are given as

$$
\begin{aligned}
A_{t}(z=0) & =\mu, \\
A_{t}\left(z=z_{h}\right) & =0, \\
f(z=0) & =1, \\
f\left(z=z_{h}\right) & =0, \\
A_{E}(z=0) & =0, \\
\phi(z=0) & =0 .
\end{aligned}
$$

Given these boundary conditions, we still have the freedom to choose the energy scale of the EMD system. We should emphasize here that at finite temperature or finite chemical potential case, the solutions here are different from those in model III. 


\section{Model V}

In model $\mathrm{V}$, we input $\phi(z)$ as

$\phi(z)=\frac{2 \sqrt{6}}{3} z \sqrt{3 d\left(3+2 d z^{2}\right)}+6 \operatorname{arcsinh}\left[\sqrt{\frac{2 d}{3}} z\right]$.

Note that the dimension of the parameter $d$ is $[E]^{2}$ and its value decides the energy scale of the EMD system. Substituting Eq. (61) into Eq. (20), we force the reality of $A_{E}(z)$ and consider the boundary condition

$$
A_{E}(z=0)=0
$$

we can derive $A_{E}(z)$ and $V_{\phi}(\phi)$ numerically. Although we cannot get the analytical form of $A_{E}(z)$, we can still derive its asymptotic expansions:

$$
\begin{aligned}
A_{E}(z \rightarrow 0)= & -\frac{8}{3} d z^{2}+\frac{8}{9} d^{2} z^{4}-\frac{512}{567} d^{3} z^{6}+\frac{1664}{1701} d^{4} z^{8} \\
& -\frac{311296}{280665} d^{5} z^{10}+\frac{19972096}{15324309} d^{6} z^{12}+\mathcal{O}\left(z^{14}\right) .
\end{aligned}
$$

As for finite temperature and finite chemical potential, the EOMs are Eqs. (14)-(17). The boundary conditions are imposed as

$$
\begin{gathered}
A_{t}(z=0)=\mu, \\
A_{t}\left(z=z_{h}\right)=0, \\
f(z=0)=1, \\
f\left(z=z_{h}\right)=0,
\end{gathered}
$$

where $z=z_{h}$ is the location of the event horizon of black hole on the coordinate $z, \mu$ is the chemical potential. Collecting these boundary conditions, the Eq. (61), and the numerical solution of $A_{E}(z)$, we can then solve the EMD system.

\section{SPECTRA OF GLUEBALLS AND ODDBALLS}

In this section, we discuss the spectra of glueballs and oddballs. There are two different methods to investigate the glueball spectra in holographic QCD. The first method can be called the "glueball fluctuations method" as in Refs. [63,94,97,109,122,135], where the scalar and tensor fluctuations are treated as the 5-dimensional fields dual to scalar and tensor glueballs respectively. Another method, which is used in this work, can be called the "glueball excitations method," where the action of the related glueball fields are introduced and the glueballs are treated as excitations from the background as used in Refs. [99,100,103,104,144], where the glueball spectra are treated in the same way as the meson spectra in the bottom-up framework. In practice, the glueball fluctuations method is more widely used in the top-down framework, and the glueball excitations method is more widely used in the bottom-up framework. We adopt the glueball excitations method in this work in order to keep the way of treating the meson spectra and glueball spectra on an equal footing in the deformed holographic QCD models.

Although we could consider more generic Lagrangians [145], we still use the simple action describing scalar, vector, and tensor glueballs/oddballs here. In the string frame, the action is

$$
\begin{aligned}
S_{g}^{s}= & -c_{g} \int \mathrm{d}^{5} x \sqrt{-g_{s}} \mathrm{e}^{-p \Phi} \\
& \times\left\{\left[\frac{1}{2} g^{s M N} \partial_{M} \mathcal{S} \partial_{N} \mathcal{S}+\frac{1}{2} \mathrm{e}^{-c_{\text {r.m. }} \Phi} M_{\mathcal{S}, 5}^{2} \mathcal{S}^{2}\right]\right. \\
& +\left[\frac{1}{4} g^{s M \tilde{M}} g^{s N \tilde{N}}\left(\partial_{M} \mathcal{V}_{N}-\partial_{N} \mathcal{V}_{M}\right)\left(\partial_{\tilde{M}} \mathcal{V}_{\tilde{N}}-\partial_{\tilde{N}} \mathcal{V}_{\tilde{M}}\right)\right. \\
& \left.+\frac{1}{2} \mathrm{e}^{-c_{\text {r.m. }} \Phi_{M}} M_{\mathcal{V}, 5}^{2} \mathcal{V}^{2}\right] \\
& +\left[\frac{1}{2} \nabla_{L} \mathcal{T}_{M N} \nabla^{L} \mathcal{T}^{M N}-\nabla_{L} \mathcal{T}^{L M} \nabla^{N} \mathcal{T}_{N M}\right. \\
& +\nabla_{M} \mathcal{T}^{M N} \nabla_{N} \mathcal{T}-\frac{1}{2} \nabla_{M} \mathcal{T} \nabla^{M} \mathcal{T} \\
& \left.+\frac{1}{2} \mathrm{e}^{-c_{\text {r.m. }} \Phi_{M}} M_{\mathcal{T}, 5}^{2}\left(\mathcal{T}^{M N} h_{M N}-\mathcal{T}^{2}\right)\right] \\
& + \text { terms for high spin fields }(\operatorname{spin} S \geqslant 3)\}
\end{aligned}
$$

where $s$ denotes the string frame, $c_{g}$ describes the coupling strength of glueballs part in the whole theory. The fields $\mathcal{S}$, $\mathcal{V}_{M}$, and $\mathcal{T}_{M N}$ are 5-dimensional fields that are dual to scalar glueball, vector glueball, and spin-2 glueball operators respectively. $\mathcal{T}=g^{s M N} \mathcal{T}_{M N}$, and $\mathcal{T}_{M N}$ satisfies the following constraints

$$
\begin{aligned}
\nabla_{M} \mathcal{T}^{M N} & =0, \quad \mathcal{T}=0, \\
\mathcal{T}_{\mu \nu} & =\frac{1}{z^{2}} \mathrm{e}^{2 A_{s}} \tilde{\mathcal{T}}_{\mu \nu}, \quad \mathcal{T}_{M z}=0 .
\end{aligned}
$$

As in Ref. [146], the parameter $p$ is introduced to make a distinction between glueballs (oddballs) with different $P$-parity:

$$
\begin{cases}p=1, & \text { for even parity } \\ p=-1, & \text { for odd parity. }\end{cases}
$$

Also we introduce a $z$ dependent modified 5-dimensional mass: 


$$
M_{5}^{2}(z)=\mathrm{e}^{-c_{\mathrm{r} . \mathrm{m} .} \Phi} M_{5}^{2},
$$

where $c_{\text {r.m. }}$ is a constant. The $M_{5}^{2}$ is listed in Table I given by the $\mathrm{AdS}_{5} / \mathrm{CFT}_{4}$ correspondence dictionary. The $\mathrm{AdS}_{5} / \mathrm{CFT}_{4}$ duality gives one-to-one correspondence between 4-dimensional operators in the $\mathcal{N}=4$ super Yang-Mills theory and the spectrum of the type IIB string theory on $\mathrm{AdS}_{5} \times S^{5}$. Based on the AdS/CFT dictionary, the conformal dimension of a $q$-form operator at the ultraviolet (UV) boundary is related to the 5-dimensional mass square $M_{5}^{2}$ of its dual field in the bulk as follows [55-57]:

$$
M_{5}^{2}=(\Delta-q)(\Delta+q-4) .
$$

\section{A. Glueballs and oddballs}

In the bottom-up holographic QCD models, one can expect a more general correspondence, i.e., each 4dimensional operator $\mathcal{O}(x)$ corresponds to a 5-dimensional field $O(x, z)$ in the bulk theory. To investigate the glueball spectra, we consider the lowest dimension operators with the corresponding quantum numbers defined in the field theory living on the 4-dimensional boundary. We show the $C$-parity even/odd glueball and oddball operators and their corresponding 5-dimensional mass square in Table I.

The lowest dimension gauge invariant three-gluon currents that couple to the exotic $0^{+-}$and $0^{--}$glueballs are constructed in Ref. [31]:

$$
j_{\alpha}^{0^{+-}}(x)=g_{s}^{3} \operatorname{Tr}\left(\left\{\left(D_{\tau} G_{\mu \nu}(x)\right),\left(D_{\tau} G_{\rho \nu}(x)\right)\right\}\left(D_{\mu} G_{\rho \alpha}(x)\right)\right),
$$

$$
j_{\alpha}^{0^{--}}(x)=g_{s}^{3} \operatorname{Tr}\left(\left\{\left(D_{\tau} G_{\mu \nu}(x)\right),\left(D_{\tau} G_{\rho \nu}(x)\right)\right\}\left(D_{\mu} \tilde{G}_{\rho \alpha}(x)\right)\right) .
$$

For trigluon glueball $1^{-+}$, and $2^{+-}$, the currents that match the unconventional quantum number and satisfy the constraints of the gauge invariance are given in Refs. [27]:

$$
\begin{aligned}
j_{\alpha}^{1^{-+}, A}(x) & =g_{s}^{3} f^{a b c} \partial_{\mu}\left[G_{\mu \nu}^{a}(x)\right]\left[G_{v \rho}^{b}(x)\right]\left[G_{\rho \alpha}^{c}(x)\right], \\
j_{\alpha}^{1^{++}, B}(x) & =g_{s}^{3} f^{a b c} \partial_{\mu}\left[G_{\mu \nu}^{a}(x)\right]\left[\tilde{G}_{v \rho}^{b}(x)\right]\left[\tilde{G}_{\rho \alpha}^{c}(x)\right], \\
j_{\alpha}^{1^{++}}, C_{(x)} & =g_{s}^{3} f^{a b c} \partial_{\mu}\left[\tilde{G}_{\mu \nu}^{a}(x)\right]\left[G_{v \rho}^{b}(x)\right]\left[\tilde{G}_{\rho \alpha}^{c}(x)\right], \\
j_{\alpha}^{1^{-+}, D}(x) & =g_{s}^{3} f^{a b c} \partial_{\mu}\left[\tilde{G}_{\mu \nu}^{a}(x)\right]\left[\tilde{G}_{v \rho}^{b}(x)\right]\left[G_{\rho \alpha}^{c}(x)\right],
\end{aligned}
$$

and

$$
\begin{aligned}
& j_{\mu \alpha}^{2^{+-}, A}(x)=g_{s}^{3} d^{a b c}\left[G_{\mu \nu}^{a}(x)\right]\left[G_{v \rho}^{b}(x)\right]\left[G_{\rho \alpha}^{c}(x)\right], \\
& j_{\mu \alpha}^{2^{+-}, B}(x)=g_{s}^{3} d^{a b c}\left[G_{\mu \nu}^{a}(x)\right]\left[\tilde{G}_{v \rho}^{b}(x)\right]\left[\tilde{G}_{\rho \alpha}^{c}(x)\right], \\
& j_{\mu \alpha}^{2^{+-}, C}(x)=g_{s}^{3} d^{a b c}\left[\tilde{G}_{\mu \nu}^{a}(x)\right]\left[G_{v \rho}^{b}(x)\right]\left[\tilde{G}_{\rho \alpha}^{c}(x)\right], \\
& j_{\mu \alpha}^{2^{+-}, D}(x)=g_{s}^{3} d^{a b c}\left[\tilde{G}_{\mu \nu}^{a}(x)\right]\left[\tilde{G}_{v \rho}^{b}(x)\right]\left[G_{\rho \alpha}^{c}(x)\right],
\end{aligned}
$$

where $d^{a b c}$ stands for the totally symmetric $S U_{c}(3)$ structure constant and $g_{\alpha \beta}^{t}=g_{\alpha \beta}-\partial_{\alpha} \partial_{\beta} / \partial^{2}$.

\section{B. Equation of motion for scalar, vector, and tensor glueballs/oddballs}

From the 5-dimensional action for the glueball/oddball in the string frame Eq. (65), we can derive the equation of motion for the glueballs. The equation of motion for the scalar glueballs $\mathcal{S}$ is given as

TABLE I. 5-dimensional mass square of $C$-parity even glueballs and $C$-parity odd oddballs. The operators are taken from Refs. [27,31,32,94].

\begin{tabular}{lcccc}
\hline \hline$J^{P C}$ & 4-dimensional operator: $\mathcal{O}(x)$ & $\Delta$ & $q$ & $M_{5}^{2}$ \\
\hline $0^{++}$ & $\operatorname{Tr}\left(G^{2}\right)=\vec{E}^{a} \cdot \vec{E}^{a}-\vec{B}^{a} \cdot \vec{B}^{a}$ & 4 & 0 & 0 \\
$0^{-+}$ & $\operatorname{Tr}(G \tilde{G})=\vec{E}^{a} \cdot \vec{B}^{a}$ & 4 & 0 & 0 \\
$0^{+-}$ & $\operatorname{Tr}\left(\left\{\left(D_{\tau} G_{\mu \nu}\right),\left(D_{\tau} G_{\rho \nu}\right)\right\}\left(D_{\mu} G_{\rho \alpha}\right)\right)$ & 9 & 0 & 45 \\
$0^{--}$ & $\operatorname{Tr}\left(\left\{\left(D_{\tau} G_{\mu \nu}\right),\left(D_{\tau} G_{\rho \nu}\right)\right\}\left(D_{\mu} \tilde{G}_{\rho \alpha}\right)\right)$ & 9 & 0 & 45 \\
$1^{-+}$ & $f^{a b c} \partial_{\mu}\left[G_{\mu \nu}^{a}\right]\left[G_{v]}^{b}\right]\left[G_{\rho \alpha}^{c}\right], f^{a b c} \partial_{\mu}\left[G_{\mu \nu}^{a}\right]\left[\tilde{G}_{v \rho}^{b}\right]\left[\tilde{G}_{\rho \alpha}^{c}\right]$, & 7 & 1 & 24 \\
& $f^{a b c} \partial_{\mu}\left[\tilde{G}_{\mu \nu}^{a}\right]\left[G_{v \rho}^{b}\right]\left[\tilde{G}_{\rho \alpha}^{c}\right], f^{a b c} \partial_{\mu}\left[\tilde{G}_{\mu \nu}^{a}\right]\left[\tilde{G}_{v \rho}^{b}\right]\left[G_{\rho \alpha}^{c}\right]$ & & & \\
$1^{+-}$ & $d^{a b c}\left(\vec{E}_{a} \cdot \vec{E}_{b}\right) \vec{B}_{c}$ & 6 & 1 & 15 \\
$1^{--}$ & $d^{a b c}\left(\vec{E}_{a} \cdot \vec{E}_{b}\right) \vec{E}_{c}$ & 6 & 1 & 15 \\
$2^{++}$ & $E_{i}^{a} E_{j}^{a}-B_{i}^{a} B_{j}^{a}-$ trace & 4 & 2 & 4 \\
$2^{-+}$ & $E_{i}^{a} B_{j}^{a}+B_{i}^{a} E_{j}^{a}-\operatorname{trace}$ & 4 & 2 & 4 \\
$2^{+-}$ & $d^{a b c} \mathcal{S}\left[E_{a}^{i}\left(\vec{E}_{b} \times \vec{B}_{c}\right)^{j}\right]$ & 6 & 2 & 16 \\
$2^{--}$ & $d^{a b c} \mathcal{S}\left[B_{a}^{i}\left(\vec{E}_{b} \times \vec{B}_{c}\right)^{j}\right]$ & 6 & 2 & 16 \\
$3^{+-}$ & $d^{a b c} \mathcal{S}\left[B_{a}^{i} B_{b}^{j} B_{c}^{k}\right]$ & 6 & 3 & 15 \\
$3^{--}$ & $d^{a b c} \mathcal{S}\left[E_{a}^{i} E_{b}^{j} E_{c}^{k}\right]$ & 6 & 3 & 15 \\
\hline \hline
\end{tabular}




$$
\begin{gathered}
-z^{3} \mathrm{e}^{-\left(3 A_{s}-p \Phi\right)} \partial_{z}\left[\frac{1}{z^{3}} \mathrm{e}^{3 A_{s}-p \Phi} \partial_{z} \mathcal{S}_{n}\right] \\
+\frac{1}{z^{2}} \mathrm{e}^{2 A_{s}} \mathrm{e}^{-c_{\text {r.m. }} \Phi} M_{\mathcal{S}, 5}^{2} \mathcal{S}_{n}=m_{\mathcal{S}, n}^{2} \mathcal{S}_{n} .
\end{gathered}
$$

Via the substitution

$$
\mathcal{S}_{n} \rightarrow z^{\frac{3}{2}} \mathrm{e}^{-\frac{1}{2}\left(3 A_{s}-p \Phi\right)} \mathcal{S}_{n}
$$

the equation can be brought into Schrödinger-like equation

$$
-\mathcal{S}_{n}^{\prime \prime}+V_{\mathcal{S}} \mathcal{S}_{n}=m_{\mathcal{G}, n}^{2} \mathcal{S}_{n}
$$

with the 5-dimensional effective Schrödinger potential

$$
\begin{aligned}
V_{\mathcal{S}}= & \frac{3 A_{s}^{\prime \prime}+\frac{3}{z^{2}}-p \Phi^{\prime \prime}}{2}+\frac{\left[3 A_{s}^{\prime}-\frac{3}{z}-p \Phi^{\prime}\right]^{2}}{4} \\
& +\frac{1}{z^{2}} e^{2 A_{s}} \mathrm{e}^{-c_{\text {r.m. }} \Phi^{\prime}} M_{\mathcal{S}, 5}^{2} \cdot
\end{aligned}
$$

The equation of motion for the vector glueballs $\mathcal{V}_{M}$ is given as

$$
\begin{aligned}
& -z \mathrm{e}^{-\left(A_{s}-p \Phi\right)} \partial_{z}\left[\frac{1}{z} \mathrm{e}^{A_{s}-p \Phi} \partial_{z} \mathcal{V}_{n}\right] \\
& +\frac{1}{z^{2}} \mathrm{e}^{2 A_{s}} \mathrm{e}^{-c_{\text {r.m. }} \Phi} M_{\mathcal{V}, 5}^{2} \mathcal{V}_{n}=m_{\mathcal{V}, n}^{2} \mathcal{V}_{n}
\end{aligned}
$$

Via the substitution

$$
\mathcal{V}_{n} \rightarrow z^{\frac{1}{2}} e^{-\frac{1}{2}\left(A_{s}-p \Phi\right)} \mathcal{V}_{n}
$$

the equation can be brought into Schrödinger-like equation

$$
-\mathcal{V}_{n}^{\prime \prime}+V_{\mathcal{V}} \mathcal{V}_{n}=m_{\mathcal{V}, n}^{2} \mathcal{V}_{n}
$$

with the 5-dimensional effective Schrödinger potential

$$
\begin{aligned}
V_{\mathcal{V}}= & \frac{A_{s}^{\prime \prime}+\frac{1}{z^{2}}-p \Phi^{\prime \prime}}{2}+\frac{\left[A_{s}^{\prime}-\frac{1}{z}-p \Phi^{\prime}\right]^{2}}{4} \\
& +\frac{1}{z^{2}} \mathrm{e}^{2 A_{s}} \mathrm{e}^{-c_{\text {r.m. }}} \Phi^{\prime} M_{\mathcal{V}, 5}^{2} .
\end{aligned}
$$

The equation of motion for the spin- 2 glueballs $\tilde{\mathcal{T}}_{M N}$ is given as

$$
\begin{aligned}
& -z^{3} \mathrm{e}^{-\left(3 A_{s}-p \Phi\right)} \partial_{z}\left[\frac{1}{z^{3}} \mathrm{e}^{3 A_{s}-p \Phi} \partial_{z} \tilde{\mathcal{T}}_{n}\right] \\
& +\frac{1}{z^{2}} \mathrm{e}^{2 A_{s}} \mathrm{e}^{-c_{\text {r.m. }} \Phi} M_{\tilde{\mathcal{T}}, 5}^{2} \tilde{\mathcal{T}}_{n}=m_{\tilde{\mathcal{T}}, n}^{2} \tilde{\mathcal{T}}_{n} .
\end{aligned}
$$

Via the substitution

$$
\tilde{\mathcal{T}}_{n} \rightarrow z^{\frac{3}{2}} \mathrm{e}^{-\frac{1}{2}\left(3 A_{s}-p \Phi\right)} \tilde{\mathcal{T}}_{n}
$$

the equation can be brought into Schrödinger-like equation

$$
-\tilde{\mathcal{T}}_{n}^{\prime \prime}+V_{\tilde{\mathcal{T}}} \tilde{\mathcal{T}}_{n}=m_{\tilde{\mathcal{T}}, n}^{2} \tilde{\mathcal{T}}_{n}
$$

with the 5-dimensional effective Schrödinger potential

$$
\begin{aligned}
V_{\tilde{\mathcal{T}}}= & \frac{3 A_{s}^{\prime \prime}+\frac{3}{z^{2}}-p \Phi^{\prime \prime}}{2}+\frac{\left[3 A_{s}^{\prime}-\frac{3}{z}-p \Phi^{\prime}\right]^{2}}{4} \\
& +\frac{1}{z^{2}} \mathrm{e}^{2 A_{s}} \mathrm{e}^{-c_{\text {r.m. }} \Phi} M_{\tilde{\mathcal{T}}, 5}^{2} .
\end{aligned}
$$

According to Ref. [74], the equation of motion for the high spin glueballs $\mathcal{H}_{M_{1} M_{2} \cdots M_{S}}$, the spin $S$ of which are larger than 2 , is given as

$$
\begin{gathered}
-z^{2 S-1} \mathrm{e}^{-\left[(2 S-1) A_{s}-p \Phi\right]} \partial_{z}\left[\frac{1}{z^{2 S-1}} \mathrm{e}^{(2 S-1) A_{s}-p \Phi} \partial_{z} \mathcal{H}_{n}\right] \\
+\frac{1}{z^{2}} \mathrm{e}^{2 A_{s}} \mathrm{e}^{-c_{\text {r.m. }} \Phi} M_{\mathcal{H}, 5}^{2} \mathcal{H}_{n}=m_{\mathcal{H}, n}^{2} \mathcal{H}_{n}
\end{gathered}
$$

where $S \geqslant 3$. Via the substitution

$$
\mathcal{H}_{n} \rightarrow z^{\frac{2 S-1}{2}} \mathrm{e}^{-\frac{1}{2}\left[(2 S-1) A_{s}-p \Phi\right]} \mathcal{H}_{n}
$$

the equation can be brought into Schrödinger-like equation

$$
-\mathcal{H}_{n}^{\prime \prime}+V_{\mathcal{H}} \mathcal{H}_{n}=m_{\mathcal{H}, n}^{2} \mathcal{H}_{n}
$$

with the 5-dimensional effective Schrödinger potential

$$
\begin{aligned}
V_{\mathcal{H}}= & \frac{(2 S-1) A_{s}^{\prime \prime}+\frac{2 S-1}{z^{2}}-p \Phi^{\prime \prime}}{2} \\
& +\frac{\left[(2 S-1) A_{s}^{\prime}-\frac{2 S-1}{z}-p \Phi^{\prime}\right]^{2}}{4} \\
& +\frac{1}{z^{2}} \mathrm{e}^{2 A_{s}} \mathrm{e}^{-c_{\text {r.m. }}} \Phi^{2} M_{\mathcal{H}, 5}^{2} .
\end{aligned}
$$

\section{Numerical results of glueballs/oddballs spectra}

We calculate the glueballs spectra using five different holographic models defined in the last section. We list the parameters used for calculating the glueballs spectra below.

\section{Model I and II}

In model I and model II, the value of the parameter is $a=0.4822 \mathrm{GeV}^{2}$. First, we do not consider the distinction between glueballs (oddballs) with different $P$-parity and do not introduce $z$ dependent modified 5-dimensional masses, that means $p=1$ for even and odd parity, and $c_{\text {r.m. }}=0$. Then we calculate the glueballs/oddballs mass spectra in model I and II, which is denoted by "Model I, II(O)" in 
TABLE II. The glueballs and oddballs mass spectra in the dynamical soft-wall model I and II without making a distinction between glueballs (oddballs) with different $P$-parity and introducing $z$ dependent modified 5-dimensional masses, compared with results from lattice QCD and QCD sum rule. The units of all the data in the table are GeV. The lattice data in the column "LQCD1," column "LQCD2," column "LQCD3," and column "LQCD4" are taken from Ref. [9], Ref [4], Ref [5], and Ref. [2] respectively. The QCD sum rule results are taken from Refs. [22,27,31,32]. Here we also list the data predicted by the single pole (SP) and dipole (DP) Regge model [51]: using the SP Regge model, the predicted mass for $2^{++}$glueball is $1.747 \mathrm{GeV}$; using the DP Regge model, the predicted masses for $2^{++}$glueball and $3^{--}$oddball are $1.758 \mathrm{GeV}$ and $3.001 \mathrm{GeV}$ respectively.

\begin{tabular}{|c|c|c|c|c|c|c|}
\hline$J^{P C}$ & LQCD1 & LQCD2 & LQCD3 & LQCD4 & QCDSR & Model I, II(O) \\
\hline $0^{++}$ & $1.653(26)$ & $1.475(30)(65)$ & $1.710(50)(80)$ & $1.730(50)(80)$ & $1.50 \pm 0.19$ & 1.876 \\
\hline $0^{*++}$ & $2.842(40)$ & $2.755(70)(120)$ & $\ldots$ & $2.670(180)(130)$ & 2.0-2.1 & 2.541 \\
\hline $0^{* *++}$ & $\ldots$ & $3.370(100)(150)$ & $\ldots$ & $\ldots$ & $\ldots$ & 3.062 \\
\hline $0^{* * *++}$ & $\ldots$ & $3.990(210)(180)$ & $\ldots$ & $\ldots$ & $\ldots$ & 3.506 \\
\hline $2^{++}$ & $2.376(32)$ & $2.150(30)(100)$ & $2.390(30)(120)$ & $2.400(25)(120)$ & $2.0 \pm 0.1$ & 7.895 \\
\hline $2^{*++}$ & $3.30(5)$ & $2.880(100)(130)$ & $\ldots$ & $\ldots$ & $2.2-2.3$ & 8.506 \\
\hline $0^{-+}$ & $2.561(40)$ & $2.250(60)(100)$ & $2.560(35)(120)$ & $2.590(40)(130)$ & $2.05 \pm 0.19$ & 1.876 \\
\hline $0^{*-+}$ & $3.54(8)$ & $3.370(150)(150)$ & $\ldots$ & $3.640(60)(180)$ & $2.1-2.3$ & 2.541 \\
\hline $1^{-+}$ & $4.12(8)$ & $\ldots$ & $\ldots$ & $\ldots$ & $\ldots$ & 18.484 \\
\hline $1^{*-+}$ & $4.16(8)$ & $\ldots$ & $\ldots$ & $\ldots$ & $\ldots$ & 19.137 \\
\hline $1^{* *-+}$ & $4.20(9)$ & $\ldots$ & $\ldots$ & $\ldots$ & $\ldots$ & 19.752 \\
\hline $2^{-+}$ & $3.07(6)$ & $2.780(50)(130)$ & $3.040(40)(150)$ & $3.100(30)(150)$ & $\ldots$ & 7.895 \\
\hline $2^{*-+}$ & $3.97(7)$ & $3.480(140)(160)$ & $\ldots$ & $3.890(40)(190)$ & $\ldots$ & 8.506 \\
\hline $0^{+-}$ & $\ldots$ & $\ldots$ & $4.780(60)(230)$ & $4.740(70)(230)$ & $9.2_{-1.4}^{+1.3}$ & 25.155 \\
\hline $1^{+-}$ & $2.944(42)$ & $2.670(65)(120)$ & $2.980(30)(140)$ & $2.940(30)(140)$ & $2.87_{-0.20}^{+0.17}$ & 14.713 \\
\hline $1^{*+-}$ & $3.80(6)$ & $\ldots$ & 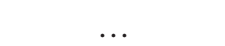 & 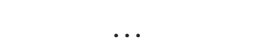 & $\ldots$ & 15.356 \\
\hline $2^{+-}$ & $4.24(8)$ & $\cdots$ & $4.230(50)(200)$ & $4.140(50)(200)$ & $\begin{array}{c}2.85_{-0.20}^{+0.16} \\
6.06 \pm 0.13\end{array}$ & 15.195 \\
\hline $3^{+-}$ & $3.53(8)$ & $3.270(90)(150)$ & $3.600(40)(170)$ & 3.550 (40) (170) & $2.78_{-0.23}^{+0.18}$ & 14.744 \\
\hline $3^{*+-}$ & $\ldots$ & $3.630(140)(160)$ & $\ldots$ & $\ldots$ & $\ldots$ & 15.388 \\
\hline $0^{--}$ & $\ldots$ & $\ldots$ & $\ldots$ & $\ldots$ & $6.8_{-1.2}^{+1.1}$ & 25.155 \\
\hline $1^{--}$ & $4.03(7)$ & $3.240(330)(150)$ & $3.830(40)(190)$ & 3.850 (50) (190) & $3.29_{-0.32}^{+1.49}$ & 14.713 \\
\hline $2^{--}$ & $3.92(9)$ & $3.660(130)(170)$ & $4.010(45)(200)$ & 3.930 (40) (190) & $3.16_{-0.23}^{+0.33}$ & 15.195 \\
\hline $2^{*--}$ & $\ldots$ & $3.740(200)(170)$ & $\ldots$ & $\ldots$ & $\ldots$ & 15.839 \\
\hline $3^{--}$ & $\ldots$ & $4.330(260)(200)$ & $4.200(45)(200)$ & 4.130 (90) (200) & $3.47_{-0.50}^{+?}$ & 14.744 \\
\hline
\end{tabular}

Table II. We find the calculation results of the masses of glueballs/oddballs, of which the 5D mass square $M_{5}^{2}$ in Table I are large, are much heavier than the lattice data. That is why we introduce a $z$-dependent modified 5-dimensional mass of glueball/oddball fields in Eq. (68). The value of the constant $c_{\text {r.m. }}$ in Eq. (68) is 0.4245 , which means

$$
M_{5}^{2}(z)=\mathrm{e}^{-0.4245 \Phi} M_{5}^{2}, \quad \text { model I, and II. }
$$

Here we briefly introduce how to determine the values of these two parameters. There are totally 24 glueballs/oddballs states in Table II. We first choose the lattice results for $N=13$ glueballs/oddballs states:

$0^{++}, \quad 0^{*++}, \quad 2^{++}, \quad 2^{*++}, 0^{-+}, 0^{*-+}$,

$2^{-+}, \quad 2^{*-+}, 1^{+-}, \quad 2^{+-}, \quad 3^{+-}, \quad 1^{--}, \quad 2^{--}$.

For the mass of every state, there are more than one lattice result. We average these lattice results for every state and then we use the least-squares method to optimize the model parameters $a$ and $c_{\text {r.m. }}$. For details, we minimize the quantity

$$
\chi^{2}\left(a, c_{\text {r.m. }}\right)=\sum_{n=1}^{N} \frac{\left[m_{i, \text { latt }}-m_{i, \text { holog }}\left(a, c_{\text {r.m. }}\right)\right]^{2}}{\sigma_{i}^{2}},
$$

where $m_{i \text {,latt }}$ is the averaged lattice result for the $i$ th glueball/ oddball state and $\sigma_{i}$ is the standard deviation of the errors of $m_{i, \text { latt }} \cdot m_{i, \text { holog }}\left(a, c_{\text {r.m. }}\right)$ is the calculated result for this state in the holographic model I and II when the value of $a$ and $c_{\text {r.m. }}$. are fixed. Here we sum for all the $N=13$ selected glueballs/ oddballs states listed in Eq. (91). After fixing the parameters, we calculate the masses for other 11 states as the predictions of the holographic models.

Following the procedure in Ref. [147], we then analyze the covariance of the parameters $a$ and $c_{\text {r.m. }}$. The uncertainties in $a$ is 


$$
\delta a=\sum_{i=1}^{N} \frac{\partial a}{\partial m_{i, \text { latt }}} \delta m_{i, \text { latt }}
$$

where $\delta m_{i, \text { latt }}$ is the uncertainties in the lattice data $m_{i \text {, latt }}$. Similarly, the uncertainties in $c_{\text {r.m. }}$ is

$$
\delta c_{\mathrm{r} . \mathrm{m} .}=\sum_{i=1}^{N} \frac{\partial c_{\mathrm{r} . \mathrm{m} .}}{\partial m_{i, \mathrm{latt}}} \delta m_{i, \mathrm{latt}} .
$$

So the covariance of the parameters $a$ and $c_{\text {r.m. }}$ is

$$
\begin{aligned}
\sigma_{\text {params }}^{2}= & \left(\begin{array}{cc}
\langle\delta a \delta a\rangle & \left\langle\delta a \delta c_{\text {r.m. }}\right\rangle \\
\left\langle\delta c_{\text {r.m. }} \delta a\right\rangle & \left\langle\delta c_{\text {r.m. }} \delta c_{\text {r.m. }}\right\rangle
\end{array}\right) \\
= & \left(\begin{array}{cc}
\sum_{i=1}^{N} \frac{\partial a}{\partial m_{i, \text { latt }}} \frac{\partial a}{\partial m_{i, \text { latt }}} \sigma_{i}^{2} & \sum_{i=1}^{N} \frac{\partial a}{\partial m_{i, \text { latt }}} \frac{\partial c_{\text {r.m. }}}{\partial m_{i, \text { latt }}} \sigma_{i}^{2} \\
\sum_{i=1}^{N} \frac{\partial a}{\partial m_{i, \text { latt }}} \frac{\partial c_{\text {r.m. }}}{\partial m_{i, \text { latt }}} \sigma_{i}^{2} & \sum_{i=1}^{N} \frac{\partial c_{\text {r.m. }} .}{\partial m_{i, \text { latt }}} \frac{\partial c_{\text {r.m. }}}{\partial m_{i, \text { latt }}} \sigma_{i}^{2}
\end{array}\right),
\end{aligned}
$$

where the symbol " \langle\rangle " represents an ensemble average and the equation

$$
\left\langle\delta m_{i, \text { latt }} \delta m_{i, \text { latt }}\right\rangle=\delta_{i j} \sigma_{i}^{2}
$$

is used. The symbol $\delta_{i j}$ in Eq. (96) is the Kronecker delta. Equation (96) is valid since we assume that the errors $\delta m_{i, \text { latt }}$ are statistically uncorrelated.

The numerical result is

$$
\sigma_{\text {params }}^{2}=\left(\begin{array}{cc}
2.0050 \mathrm{GeV}^{4} & 1.9844 \mathrm{GeV}^{2} \\
1.9844 \mathrm{GeV}^{2} & 3.1267
\end{array}\right) \times 10^{-4}
$$

By using a similar method, we can calculate the covariance of the holographic results for the glueballs/oddballs spectra. The holographic results for the glueballs/oddballs spectra and the standard deviations of their errors are denoted by "Model I, II" in Table III.

Note that the 5-dimensional field $\Phi$ and $\phi$ are different, the relationship between them is Eq. (8):

$$
\phi=\sqrt{\frac{8}{3}} \Phi
$$

\begin{tabular}{|c|c|c|c|c|c|c|c|}
\hline$J^{P C}$ & LQCD1-4 & QCDSR & EHM & Model I, II & Model III, IV(1) & Model III, IV(2) & Model V \\
\hline $0^{++}$ & $1.475(30)(65)-1.730(50)(80)$ & $1.50 \pm 0.19$ & 1.475 & $1.876(28)$ & $1.545(20)$ & 1.593 & $1.954(28)$ \\
\hline $0^{*++}$ & $2.670(180)(130)-2.842(40)$ & $2.0-2.1$ & 2.755 & $2.541(37)$ & $2.539(32)$ & 2.618 & $2.498(35)$ \\
\hline $0^{* *++}$ & $3.370(100)(150)$ & $\ldots$ & 3.376 & $3.062(45)$ & $3.211(41)$ & 3.311 & $2.944(42)$ \\
\hline $0^{* * *++}$ & $3.990(210)(180)$ & $\ldots$ & 3.891 & $3.506(51)$ & $3.760(48)$ & 3.877 & $3.330(47)$ \\
\hline $2^{++}$ & $2.150(30)(100)-2.400(25)(120)$ & $2.0 \pm 0.1$ & 2.180 & $2.689(28)$ & $2.459(26)$ & 2.203 & $2.755(27)$ \\
\hline $2^{*++}$ & $2.880(100)(130)-3.30(5)$ & $2.2-2.3$ & 2.899 & $3.208(29)$ & $3.088(25)$ & 3.006 & $3.195(26)$ \\
\hline $0^{-+}$ & $2.250(60)(100)-2.590(40)(130)$ & $2.05 \pm 0.19$ & $\ldots$ & $2.323(34)$ & $2.527(32)$ & 2.606 & $2.268(32)$ \\
\hline $0^{*-+}$ & $3.370(150)(150)-3.640(60)(180)$ & $2.1-2.3$ & $\ldots$ & $2.932(43)$ & $3.217(41)$ & 3.317 & $2.798(40)$ \\
\hline $1^{-+}$ & $4.12(8)$ & $\ldots$ & $\ldots$ & $3.637(36)$ & $3.920(36)$ & 3.588 & $3.566(34)$ \\
\hline $1^{*-+}$ & $4.16(8)$ & $\ldots$ & $\ldots$ & $4.126(37)$ & $4.479(36)$ & 4.221 & $3.990(33)$ \\
\hline $1^{* *-+}$ & $4.20(9)$ & $\ldots$ & $\ldots$ & $4.538(41)$ & $4.943(39)$ & 4.730 & $4.353(35)$ \\
\hline $2^{-+}$ & $2.780(50)(130)-3.100(30)(150)$ & $\ldots$ & $\ldots$ & $3.216(29)$ & $3.306(26)$ & 3.161 & $3.166(27)$ \\
\hline $2^{*-+}$ & $3.480(140)(160)-3.97(7)$ & $\ldots$ & $\ldots$ & $3.658(33)$ & $3.737(34)$ & 3.703 & $3.558(29)$ \\
\hline $0^{+-}$ & $4.740(70)(230)-4.780(60)(230)$ & $9.2_{-1.4}^{+1.3}$ & $\ldots$ & $3.428(41)$ & $3.632(45)$ & 3.165 & $3.420(40)$ \\
\hline $1^{+-}$ & $2.670(65)(120)-2.980(30)(140)$ & $2.87_{-0.20}^{+0.17}$ & $\cdots$ & $3.216(35)$ & $3.336(38)$ & 2.954 & $3.212(34)$ \\
\hline $1^{*+-}$ & $3.80(6)$ & $\ldots$ & $\ldots$ & $3.735(34)$ & $3.926(33)$ & 3.652 & $3.655(31)$ \\
\hline $2^{+-}$ & $4.140(50)(200)-4.24(8)$ & $\begin{array}{c}2.85_{-0.20}^{+0.16} \\
6.06 \pm 0.13\end{array}$ & $\begin{array}{l}\cdots \\
\cdots\end{array}$ & $3.131(37)$ & $3.209(41)$ & 2.786 & $3.147(35)$ \\
\hline $3^{+-}$ & $3.270(90)(150)-3.600(40)(170)$ & $2.78_{-0.23}^{+0.18}$ & $\ldots$ & $3.007(37)$ & $3.025(44)$ & 2.572 & $3.047(36)$ \\
\hline $3^{*+-}$ & $3.630(140)(160)$ & $\ldots$ & $\ldots$ & $3.555(34)$ & $3.668(34)$ & 3.369 & $3.510(31)$ \\
\hline $0^{--}$ & $\ldots$ & $6.8_{-1.1}^{+1.1}$ & $\ldots$ & $3.890(38)$ & $4.249(38)$ & 3.907 & $3.795(36)$ \\
\hline $1^{--}$ & $3.240(330)(150)-4.03(7)$ & $3.29_{-0.32}^{+1.49}$ & $\ldots$ & $3.508(34)$ & $3.746(34)$ & 3.441 & $3.446(32)$ \\
\hline $2^{--}$ & $3.660(130)(170)-4.010(45)(200)$ & $3.16_{-0.23}^{+0.33}$ & $\ldots$ & $3.621(34)$ & $3.903(33)$ & 3.619 & $3.539(32)$ \\
\hline $2^{*--}$ & $3.740(200)(170)$ & $\ldots$ & $\ldots$ & $4.093(37)$ & $4.426(35)$ & 4.211 & $3.951(32)$ \\
\hline $3^{--}$ & $4.130(90)(200)-4.330(260)(200)$ & $3.47_{-0.50}^{+?}$ & $\ldots$ & $3.700(34)$ & $4.017(33)$ & 3.765 & $3.600(32)$ \\
\hline
\end{tabular}

TABLE III. The glueballs and oddballs mass spectra in the dynamical soft-wall model, compared with results from lattice QCD and QCD sum rule. The units of all the data in the table are GeV. The lattice data is taken from Refs. [2,4,5,9]. The QCD sum rule results are taken from Refs. [22,27,31,32]. The data in the column labeled by EHM is the result of an effective holographic model from Ref. [109] by using the glueball fluctuations method. Here we also list the data predicted by the SP and DP Regge model [51]: using the SP Regge model, the predicted mass for $2^{++}$glueball is $1.747 \mathrm{GeV}$; using the DP Regge model, the predicted masses for $2^{++}$glueball and $3^{--}$ oddball are $1.758 \mathrm{GeV}$ and $3.001 \mathrm{GeV}$ respectively. 


\section{Model III and IV}

In model III and model IV, the value of the parameter is $b=1.5360 \mathrm{GeV}^{2}$. The value of the constant $c_{\text {r.m. }}$. in Eq. (68) is 0.4593 , which means

$$
M_{5}(z)^{2}=\mathrm{e}^{-0.4593 \Phi} M_{5}^{2}, \quad \text { model III and IV }
$$

The covariance of the parameters $b$ and $c_{\text {r.m. }}$ is

$$
\begin{aligned}
& \sigma_{\text {params }}^{2}=\left(\begin{array}{cc}
\langle\delta b \delta b\rangle & \left\langle\delta b \delta c_{\text {r.m. }}\right\rangle \\
\left\langle\delta c_{\text {r.m. }} \delta b\right\rangle & \left\langle\delta c_{\text {r.m. }} \delta c_{\text {r.m. }}\right\rangle
\end{array}\right) \\
& =\left(\begin{array}{cc}
15.3217 \mathrm{GeV}^{4} & 6.1579 \mathrm{GeV}^{2} \\
6.1579 \mathrm{GeV}^{2} & 4.0296
\end{array}\right) \times 10^{-4} .
\end{aligned}
$$

The method to calculate the value of the parameters $b, c_{\text {r.m. }}$. and the covariance of the parameters $\sigma_{\text {params }}^{2}$ are similar to that in subsubsection IV C 1.

The holographic results for the glueballs/oddballs spectra and the standard deviations of their errors are denoted by "Model III, IV(1)" in Table III.

In Ref. [146], the authors also use model III to calculate the glueballs spectra. There they use the parameters $b=$ $\frac{2 \sqrt{6}}{3} \mathrm{GeV}^{2}[148]$ and $c_{\text {r.m. }}=\frac{2}{3}$. We also calculate the glueballs spectra using these values of parameters and list the results denoted by Model III, IV(2) in the Table III.

\section{Model V}

In model $\mathrm{V}$, the value of the parameter is $d=0.2463 \mathrm{GeV}^{2}$. The value of the constant $c_{\text {r.m. }}$ in Eq. (68) is 0.3576 , which means

$$
M_{5}(z)^{2}=\mathrm{e}^{-0.3576 \Phi} M_{5}^{2}, \quad \text { model V }
$$

The covariance of the parameters $d$ and $c_{\text {r.m. }}$ is

$$
\begin{aligned}
\sigma_{\text {params }}^{2} & =\left(\begin{array}{cc}
\langle\delta d \delta d\rangle & \left\langle\delta d \delta c_{\text {r.m. }}\right\rangle \\
\left\langle\delta c_{\text {r.m. }} \delta d\right\rangle & \left\langle\delta c_{\text {r.m. } .} \delta c_{\text {r.m. }}\right\rangle
\end{array}\right) \\
& =\left(\begin{array}{cc}
0.4898 \mathrm{GeV}^{4} & 0.9016 \mathrm{GeV}^{2} \\
0.9016 \mathrm{GeV}^{2} & 2.4450
\end{array}\right) \times 10^{-4}
\end{aligned}
$$

The method to calculate the value of the parameters $d, c_{\text {r.m. }}$ and the covariance of the parameters $\sigma_{\text {params }}^{2}$ are similar to that in Sec. IV C 1.

The holographic results for the glueballs/oddballs spectra and the standard deviations of their errors are denoted by "Model V" in Table III.

The corresponding results for glueballs and oddballs spectra are also shown in Fig. 2 and Fig. 3, respectively.

\section{Compare results with lattice $Q C D, Q C D$ sum rule and pp high energy scattering}

We summarize our holographic results of glueballs/oddballs spectra and then compare them with the results from lattice simulation and QCD sum rule in Table III. We also list the result of an effective holographic model, which is taken from [109] and is consistent with the data from lattice QCD, where the authors treat the scalar and tensor fluctuations in the gravity-dilaton action as the 5-dimensional fields that dual to scalar and tensor glueballs respectively, i.e., the glueball fluctuations method as we mentioned at the beginning of Sec. IV. To explicitly see the difference between results from holographic QCD models and those from lattice simulation, we also list results in Fig. 2 for $C$-parity even glueballs, and in Fig. 3 for $C$-parity odd oddballs.

In the framework of holography, the states $J^{P C}$ with the same angular momentum $J$ and the same $C$-parity corresponds to different operators, however, the dimensions of which are the same. Thus, they have the same dimension and 5-dimensional mass, and the mass splitting for different $P$-parity states is realized by $\mathrm{e}^{-p \Phi}$ in Eq. (65). The states $J^{P C}$ with the same angular momentum $J$ and the same $P$-parity but different $C$-parity have different operators, the dimensions of which are also different. Thus, they have different 5-dimensional masses, which naturally induces the mass splitting for different $C$-parity states. From the results in Table III, Figs. 2, and 3, we can see that with only 2 parameters, the model predictions on glueballs/oddballs spectra in general are in good agreement with lattice results except three oddballs states $0^{+-}, 2^{+-}$, and $3^{--}$. Here we also would like to mention that the data predicted by the single pole (SP) and dipole (DP) Regge model [51] to fit the high energy $p p$ scattering: using the SP Regge model, the predicted mass for $2^{++}$glueball is $1.747 \mathrm{GeV}$; using the DP Regge model, the predicted masses for $2^{++}$glueball and $3^{--}$oddball are $1.758 \mathrm{GeV}$ and $3.001 \mathrm{GeV}$, respectively. These predicted values are a little bit lower than the results predicted from holography but still in reasonable regions. It might indicate that the mass $1.747 \mathrm{GeV} / 1.758 \mathrm{GeV} 2^{++}$ glueball and mass $3.001 \mathrm{GeV}^{--}$oddball are hybrid glueball/oddball states mixing with quark states.

\section{EQUATION OF STATE}

Thermodynamic properties of the Yang-Mills theory has been investigated in the holographic frame [63,65]. Here, with parameters used to calculate the glueballs/oddballs spectra listed in Table III, we check the corresponding thermodynamic properties of the system in our holographic models.

\section{A. Model I and II}

In model I and model II, the value of the parameters are $a=0.4822 \mathrm{GeV}^{2}$, and the 5-dimensional Newtown constant $G_{5}=1$. Then we numerically calculate the thermodynamic 


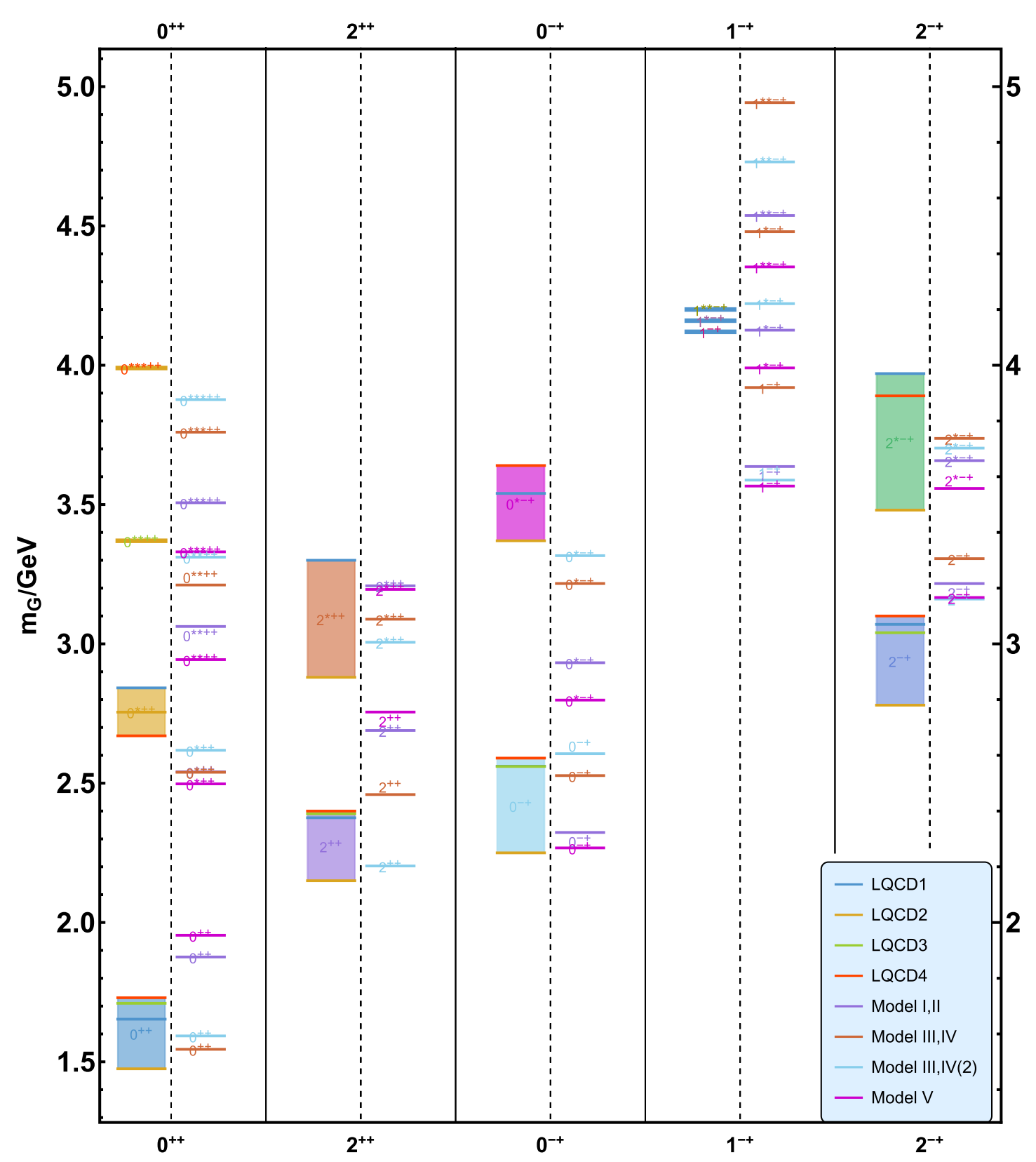

FIG. 2. The mass spectra of $J^{P C}(C=1)$ glueballs in the dynamical soft-wall model, compared with lattice data. This figure are split into five panels, that are divided by black solid lines. From left to right, the mass data in these panels belong to $0^{++}$states, $2^{++}$states, $0^{-+}$states, $1^{-+}$states, and $2^{-+}$states respectively. In every panel, the black dashed line split it into two parts. The left one contains lattice data taken from Refs. [2,4,5,9]. The steel blue lines, goldenrod lines, olive drab lines, orange red lines are lattice data taken from Ref. [9], Ref [4], Ref [5], and Ref [2] respectively. The minimal value and maximal value of a set of discrete data that belongs to the same glueball state decide the positions of lower and upper bound of the bar in the figure respectively. The data in the right part are calculated in our holographic models. The medium purple lines, sienna lines, sky blue lines, and magenta lines are results from Model I, II, Model III, IV (1), Model III, IV(2), and Model V, respectively.

properties in model I and model II. In model II, we utilize the numerical method in Refs. $[133,149]$ to investigate the thermodynamic properties. The results are different for these two models, as we emphasized in Sec. III C 1. The deconfined temperature $T_{c}=480.956 \mathrm{MeV}$ for model I with inputting $A_{E}(z)$ and $T_{c}=465.924 \mathrm{MeV}$ for model II with inputting $V_{\phi}(\phi)$. We plot the thermodynamical quantities in Fig. 4. The red points with error bar are lattice simulation of $S U$ (3) YangMills results in Ref. [150].

It is noticed that even though model I and model II can describe glueballs/oddballs spectra, the corresponding thermodynamic properties shown in Fig. 4 are not in good agreement with lattice results [150] for the pure gluon system. From the asymptotic analysis of the dilaton field at 


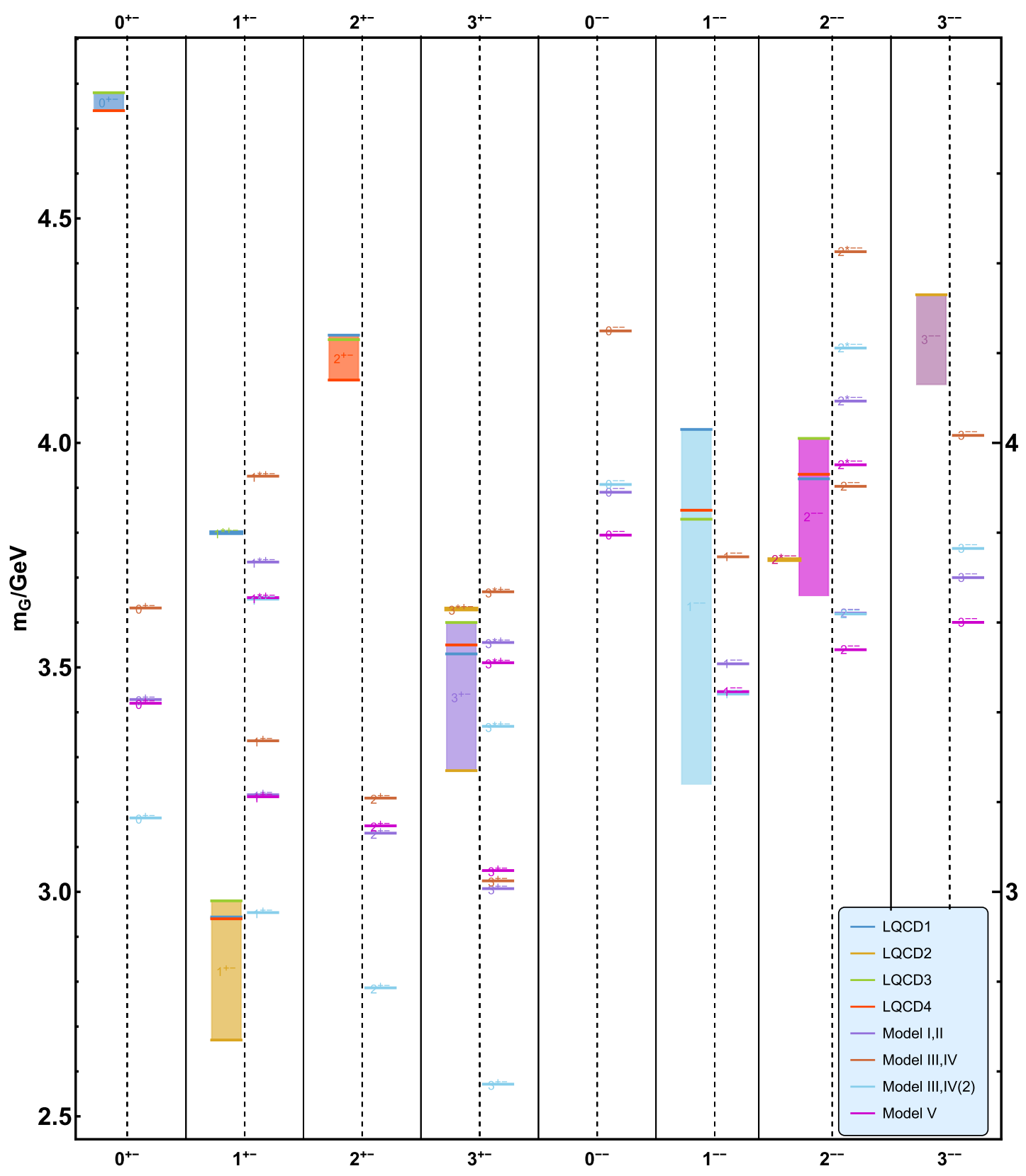

FIG. 3. The mass spectra of $J^{P C}(C=-1)$ oddballs in the dynamical soft-wall model, compared with lattice data. This figure are split into eight panels, that are divided by black solid lines. From left to right, the mass data in these panels belong to $0^{+-}$states, $1^{+-}$states, $2^{+-}$states, $3^{+-}$states, $0^{--}$states, $1^{--}$states, $2^{--}$states, and $3^{--}$states respectively. In every panel, the black dashed line split it into two parts. The left one contains lattice data taken from Refs. [2,4,5,9]. The steel blue lines, goldenrod lines, olive drab lines, orange red lines are lattice data taken from Ref. [9], Ref. [4], Ref. [5], and Ref. [2], respectively. The minimal value and maximal value of a set of discrete data that belongs to the same oddball state decide the positions of lower and upper bound of the bar in the figure, respectively. The data in the right part are calculated in our holographic models. The medium purple lines, sienna lines, sky blue lines, and magenta lines are results from Model I, II, Model III, IV(1), Model III, IV(2), and Model V, respectively.

UV boundary Eq. (27) in Sec. III A, we can see that the leading order of the 5-dimensional dilaton field is a term proportional to $z$, and the subleading order is a term proportional to $z^{3}$. So we expect the thermodynamic properties of model I and II behaves more like quark matter. We fix the value of the parameter $a$ and tune the value of $G_{5}=1$ to $G_{5}=0.42$ to meet the degrees of freedom of quark matter. In this case, the critical temperatures remain unchanged. It is found that the equation of state calculated in model I and II are qualitatively consistent 

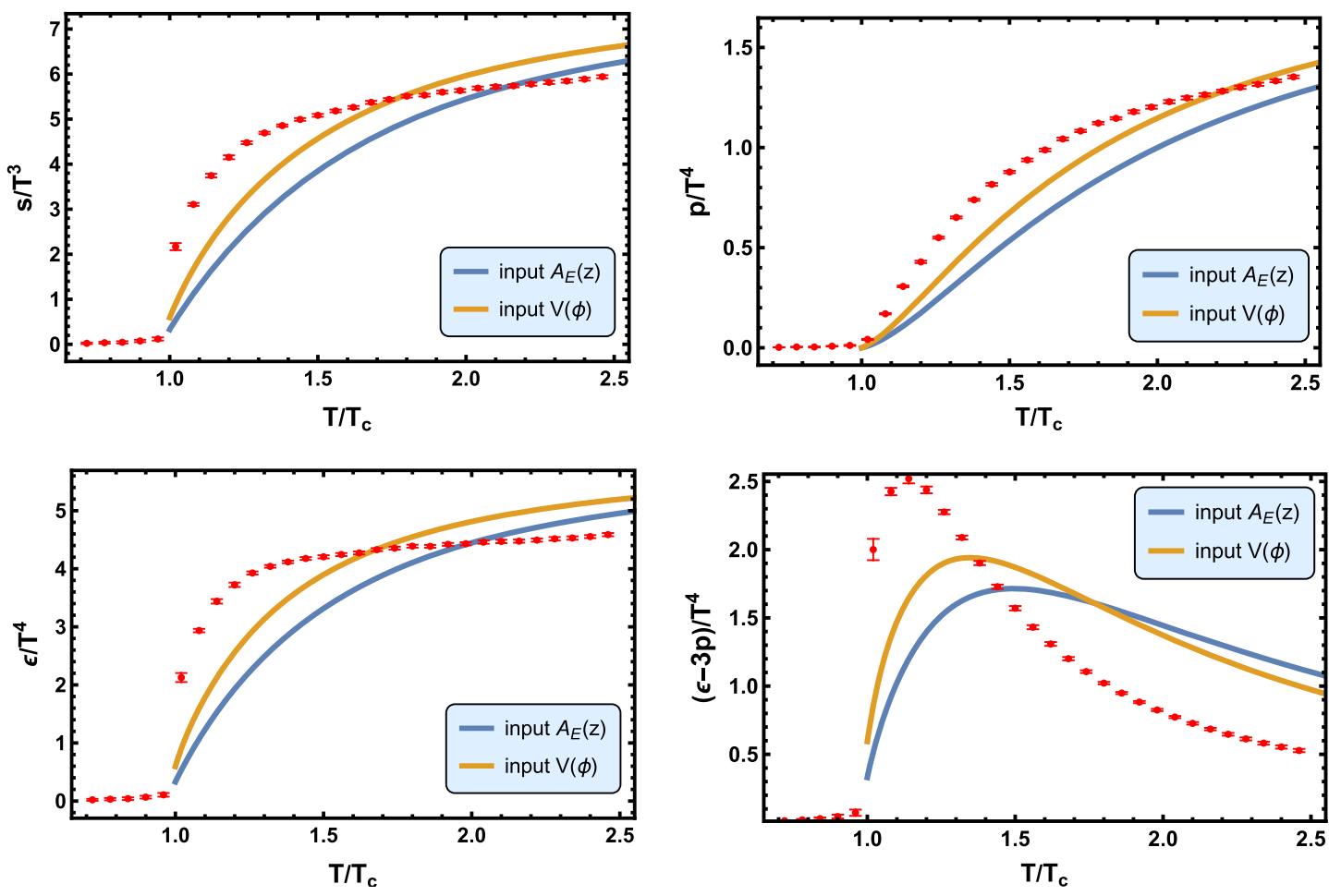

FIG. 4. The results of equation of state from model I and model II with $a=0.4822 \mathrm{GeV}^{2}$ and $G_{5}=1$. The results of the entropy density over cubic temperature (upper left panel), the pressure over quartic temperature (upper right panel), the energy density over quartic temperature (lower left panel) and the trace anomaly over quartic temperature (lower right panel) as functions of the scaled temperature $T / T_{c}$ in model I and model II, respectively. The blue line is the result for model I with inputting $A_{E}(z)$, the orange line is result for model II with inputting $V_{\phi}(\phi)$. The red points are $S U(3)$ lattice data taken from Ref. [150].

with the $2+1$ flavors lattice results in Ref. [151]. We plot the equation of state in Fig. 5. The red points with error bar are lattice simulations of $S U(3)$ equation of state taken from Ref. [150] for pure gluon system. The purple points with error bar are lattice simulations of $N_{f}=2+1$ QCD equation of state taken from Ref. [151].

\section{B. Model III and IV}

We also check the corresponding thermodynamic properties of model III and model IV. In model IIII, we use two sets of values of the parameters. The parameters $\mathcal{A}$ are $b=1.5360 \mathrm{GeV}^{2}$, and the 5-dimensional Newtown constant $G_{5}=1.35$; the parameters $\mathcal{B}$ are $b=\frac{2 \sqrt{6}}{3} \mathrm{GeV}^{2}$ as in [146], as we mention in Sec. IV C 2, the 5-dimensional Newtown constant $G_{5}=1.35$. Again, we employ the numerical method in Refs. [133,149] to investigate the thermodynamic properties in model IV. We fix the values of the characteristic energy scale [152] of the EMD system $\Lambda$ and the 5-dimensional Newtown constant $G_{5}: \Lambda=1 \mathrm{GeV}$, and $G_{5}=1.35$. Then we numerically calculate the equation of state for these two models respectively. The results are actually different for the two models, as we emphasized in Sec. III C 2. In model III, the deconfined temperature $T_{c}=$ $343.455 \mathrm{MeV}$ for parameters $\mathcal{A}$ with $b=1.5360 \mathrm{GeV}^{2}$ and $T_{c}=354.131 \mathrm{MeV}$ for parameters $\mathcal{B}$ with $b=\frac{2 \sqrt{6}}{3} \mathrm{GeV}^{2}$.
The deconfined temperature $T_{c}=269.371 \mathrm{MeV}$ in model IV with $\Lambda=1 \mathrm{GeV}$. We plot the equation of state in Fig. 6. The red points with error bar are lattice simulation of $S U(3)$ equation of state for pure gluon system in Ref. [150].

We can see from Fig. 6 that the lines for parameters $\mathcal{A}$ and parameters $\mathcal{B}$ in model III are totally the same with each other. That is not surprising because all the quantities are dimensionless in this plot.

\section{Model V}

In model $\mathrm{V}$, we use the parameters $d=0.2463 \mathrm{GeV}^{2}$, and the 5-dimensional Newtown constant $G_{5}=\frac{10}{11}$. Then we numerically calculate the equation of state. The deconfined temperature $T_{c}=522.489 \mathrm{MeV}$. We plot the equation of state in Fig. 7. The red points with error bar are lattice simulation of $S U(3)$ equation of state from Ref. [150].

If we fix the value of the parameter $d$ and tune the value of $G_{5}$ to $G_{5}=0.39$, the critical temperature remains unchanged. However, the equation of state in model $\mathrm{V}$ will be qualitatively consistent with the $2+1$ flavors lattice results, which is taken from Ref. [151]. We plot the equation of state in Fig. 8. The red points with error bar are lattice simulations of $S U(3)$ equation of state taken from Ref. [150]. The purple points with error bar are lattice simulations of $N_{f}=2+1$ QCD equation of state taken from Ref. [151]. 

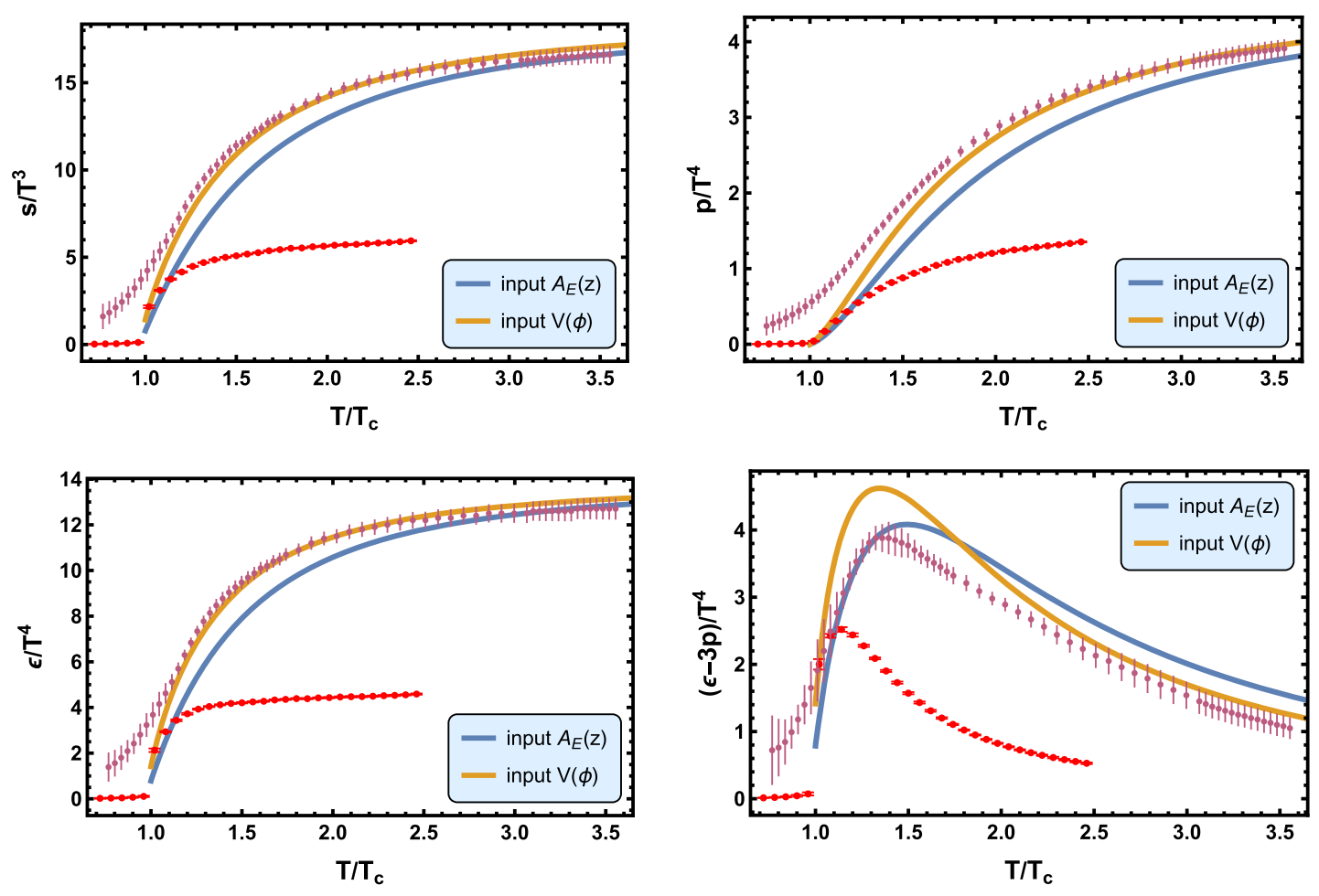

FIG. 5. The results of equation of state from model I and model II with $a=0.4822 \mathrm{GeV}^{2}$ and $G_{5}=0.42$. Upper left panel: the ratio of entropy density over cubic temperature as function of scaled temperature $T / T_{c}$. Upper right panel: the ratio of pressure over quartic temperature as function of scaled temperature $T / T_{c}$. Lower left panel: the energy density over quartic temperature as function of scaled temperature $T / T_{c}$. Lower right panel: the trace anomaly over quartic temperature as function of scaled temperature $T / T_{c}$. The blue line is for model I with inputting $A_{E}(z)$. The orange line is for model II with inputting $V_{\phi}(\phi)$. The red points are $S U(3)$ lattice data taken from Ref. [150], and the purple points are $N_{f}=2+1$ lattice data taken from Ref. [151].

The equation of state calculated in the Einstein-Maxwelldilaton model in the holographic frame can be compared not only with the lattice results for the pure gluon system, but also with the lattice results for the $2+1$ flavor system $[133,149,153]$. As the conclusion of this section, we now explain why we compare some of our holographic results with those from lattice simulation of $N_{f}=2+1$ QCD equation of state.

We start from the probe limit. The total action of 5dimensional holographic QCD model including glueball/ oddball excitations is

$$
S_{\text {total }}^{s}=S_{b}^{s}+S_{g}^{s}
$$

where $S_{b}^{s}$ is the action for the background in the string frame, and $S_{g}^{s}$ is the action describing the glueballs in the string frame. In principle, we should consider the whole action and derive the EOMs, in which the fields $\Phi, A_{\mu}, A_{s}$, and $f(z)$ are coupled with the matter fields. Thus, not only the background affects the EOMs of the matter fields, but also the matter fields provide back-reaction on the background. However, solving the fully coupled EOMs are very difficult and we are still struggling to do that. So an approximation called the probe limit is widely adopted in the literature. In the probe limit, we first neglect the coupling between the background and the matter part, and then we solve the EOMs of the background. After deriving the background, we can solve the EOMs of the matter fields that live on the background. In this procedure, we neglect the backreaction provided by the matter field on the background, as we do in this work. Thus, in the probe limit, the thermodynamic properties are entirely determined by the background. Correspondingly the dilaton field can be solved self-consistently, then from AdS/CFT dictionary, one can read the particle information of the dilaton field.

Because we neglect the back-reaction, which means the effect of the matter fields cannot be contained in the background naturally, we input different $V_{\phi}(\phi)$ (or $A_{E}$, or $\phi$ ) to produce different background solutions that are used to mimic different 4-dimensional field theories, such as pure gluon system, or $N_{f}=2+1$ QCD. To mimic different 4-dimensional field theories, the key point is to choose the appropriate value of dimension $\Delta_{\phi}$. We will explain this in the following.

Considering the flavored QCD, to describe the meson sector, the typical 5-dimensional action in bottom-up holographic QCD can be written as [72] 

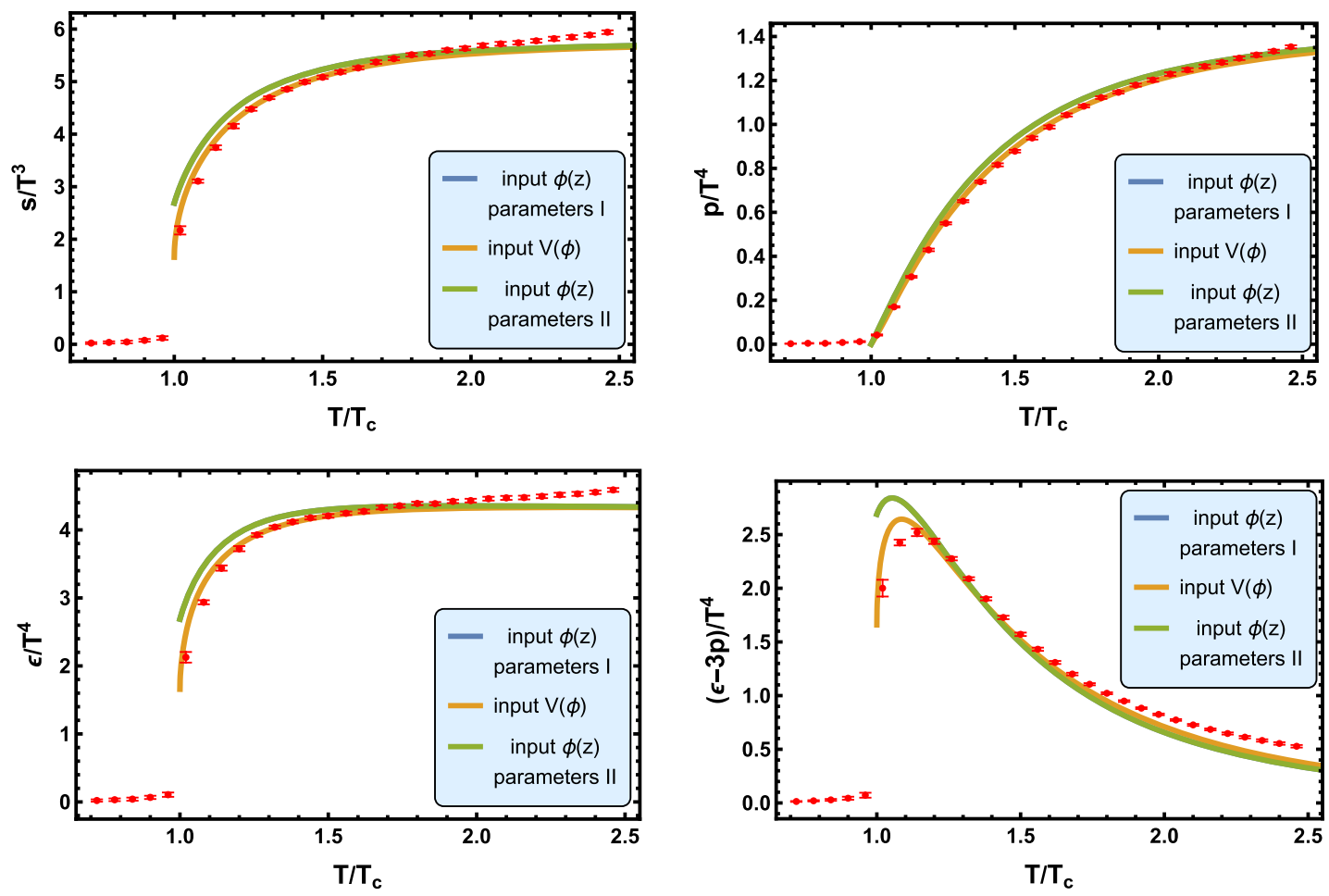

FIG. 6. The results of equation of state from model III and model IV. Upper left panel: the ratio of entropy density over cubic temperature as function of scaled temperature $T / T_{c}$. Upper right panel: the ratio of pressure over quartic temperature as function of scaled temperature $T / T_{c}$. Lower left panel: the energy density over quartic temperature as function of scaled temperature $T / T_{c}$. Lower right panel: The trace anomaly over quartic temperature as function of scaled temperature $T / T_{c}$. The blue line is for parameters $\mathcal{A}$ : $b=1.5360 \mathrm{GeV}^{2}$, and $G_{5}=1.35 \mathrm{in}$ model III, in which we input $\phi(z)$. The green line is for parameters $\mathcal{B}: b=\frac{2 \sqrt{6}}{3} \mathrm{GeV}^{2}$, and $G_{5}=1.35$ in model III. The orange line is for model IV, in which we input $V_{\phi}(\phi)$ and the parameters are $\Lambda=1 \mathrm{GeV}$, and $G_{5}=1.35$. The red points is $S U(3)$ lattice data taken from Ref. [150] for pure gluon system. The positions of the blue line and the green line are totally the same in each panel.

$$
\begin{aligned}
S_{M}= & \frac{1}{k} \int d^{5} x \sqrt{g} \mathrm{e}^{-\Phi(z)} \operatorname{Tr}\left\{|D X|^{2}-m_{5}^{2}(z)|X|^{2}\right. \\
& \left.-\lambda|X|^{4}-\frac{1}{4 g_{5}^{2}}\left(F_{L}^{2}+F_{R}^{2}\right)\right\},
\end{aligned}
$$

where $D^{M} X=\partial^{M} X-\mathrm{i} A_{L}^{M} X+\mathrm{i} X A_{R}^{M}, \quad F_{L, R}^{M N}=\partial^{M} A_{L, R}^{N}-$ $\partial^{N} A_{L, R}^{M}-\mathrm{i}\left[A_{L, R}^{M}, A_{L, R}^{N}\right], \quad A_{L}^{M}=A_{L}^{a, M} t_{L}^{a}, \quad A_{R}^{M}=A_{R}^{a, M} t_{R}^{a}, \quad t_{L}^{a}$ and $t_{R}^{a}$ are the generators of $\mathrm{SU}\left(N_{f}\right)_{L}$ and $\mathrm{SU}\left(N_{f}\right)_{R}$ respectively, and $\Phi(z)$ is the dilaton field. The dimension of the scalar field $X$ is $\Delta_{X}=3$. This leads to the bulk scalar VEV has the following behavior in the UV region:

$$
\chi(z \sim 0)=m_{q} \zeta z+\frac{\sigma}{\zeta} z^{3}+\cdots .
$$

According to the AdS/CFT dictionary, $m_{q}$ is the current quark mass, $\sigma$ is the chiral condensate, and $\zeta$ is a normalization constant.

In the fully coupled consideration, where the backreaction is taken into account, the asymptotic behavior of $\chi(z)$ guarantees the appearance of the term proportional to $z$ in the UV asymptotic expansion of $\Phi$. This is true because $\Phi$ and $\chi$ are coupled together in the fully coupled consideration.

Now we get an important conclusion: to describe the flavored QCD, there should be a term proportional to $z$ in the UV asymptotic expansion of $\Phi$. But please keep in mind that here we adopt the probe limit and use the background without backreaction to mimic the flavored QCD. Thus, taking the profile $\Delta_{\Phi}=3$ is a natural way to produce the term proportional to $z$.

In conclusion, we adopt the probe limit in the work. Although we solve the background without considering the back-reaction of the matter field, we can still use the background to mimic different 4-dimensional field theories. To mimic the flavored QCD, we should take the profile $\Delta_{\Phi}=3$. Of course, we can choose another different value of $\Delta_{\Phi}$ to mimic the pure gluon system, which is $\Delta_{\Phi}=2$ in this work.

\section{CONCLUSION AND DISCUSSION}

In this work, we study scalar, vector, and tensor glueballs/ oddballs spectra in the framework of 5-dimensional dynamical 

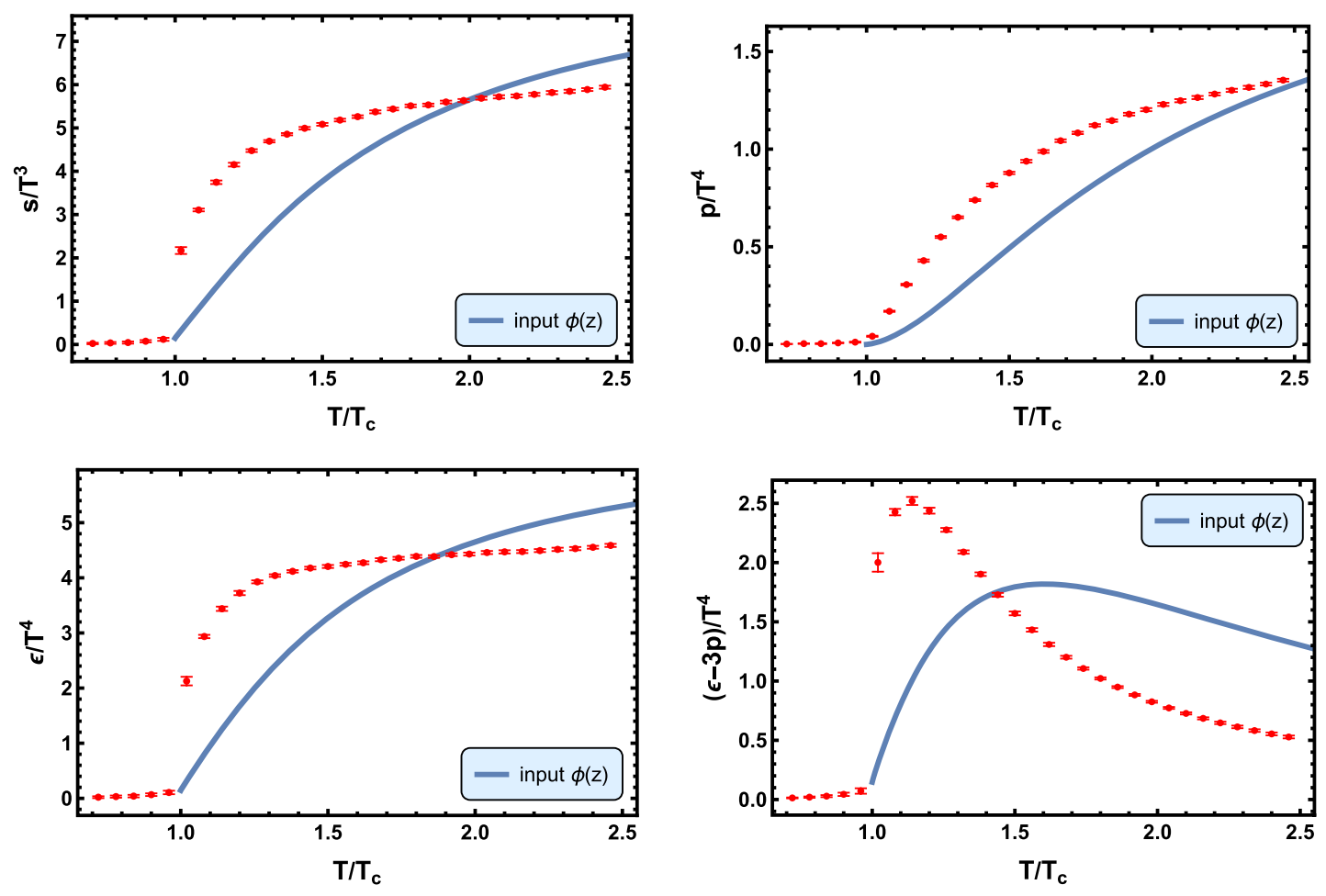

FIG. 7. The results of equation of state from model V. Upper left panel: the ratio of entropy density over cubic temperature as function of scaled temperature $T / T_{c}$. Upper right panel: the ratio of pressure over quartic temperature as function of scaled temperature $T / T_{c}$. Lower left panel: the energy density over quartic temperature as function of scaled temperature $T / T_{c}$. Lower right panel: the trace anomaly over quartic temperature as function of scaled temperature $T / T_{c}$. The blue line is for model $\mathrm{V}$, in which we input $\phi(z)$ and the parameters are $d=0.2463 \mathrm{GeV}^{2}$, and $G_{5}=\frac{10}{11}$. The red points are $S U(3)$ lattice data taken from Ref. [150] for pure gluon system.

holographic QCD model, where the metric structure is deformed self-consistently by the dilaton field. In the framework of holography, the states $J^{P C}$ with the same angular momentum $J$ and the same $C$-parity corresponds to different operators, however, the dimensions of which are the same. Thus, the corresponding 5-dimensional masses of these states are also the same, and the mass splitting for different $P$-parity states is realized by $\mathrm{e}^{-p \Phi}$ in Eq. (65). The states $J^{P C}$ with the same angular momentum $J$ and the same $P$-parity but different $C$-parity have different operators, the dimensions of which are also different. Thus, they have different 5-dimensional masses, which naturally induces the mass splitting for different $C$-parity states.

From the results in Table III, Figs. 2, and 3, we can see that with only two parameters, the model predictions on glueballs/oddballs spectra in general are in good agreement with lattice results except three oddballs states $0^{+-}, 2^{+-}$ and $3^{--}$. Here we also would like to mention that the data predicted by the SP and DP Regge model [51] to fit the high energy $p p$ scattering: using the SP Regge model, the predicted mass for $2^{++}$glueball is $1.747 \mathrm{GeV}$; using the DP Regge model, the predicted masses for $2^{++}$glueball and $3^{--}$oddball are $1.758 \mathrm{GeV}$ and $3.001 \mathrm{GeV}$ respectively. These predicted values are a little bit lower than the results predicted from holography but still in reasonable regions. It might indicate that the mass $1.747 \mathrm{GeV} / 1.758 \mathrm{GeV} 2^{++}$ glueball and mass $3.001 \mathrm{GeV} 3^{--}$oddball are hybrid glueball/oddball states mixing with quark states.

From the results of glueballs/oddballs spectra at zero temperature and zero density and the equation of state at finite temperature, we obtain the following conclusions. (1) For the same set of vacuum solutions to the Einstein field equations and the equation of motion of the dilaton field $\phi(z)$, inputting the function $A_{E}(z)$ and inputting the dilaton potential $V_{\phi}(\phi)$ give the different equation of state indeed. (2) The model with quadratic dilaton field $\phi(z)$ can simultaneously describe glueballs/oddballs spectra as well as the equation of state of pure gluon system. The model with quadratic $A_{E}(z)$ can describe glueballs/oddballs spectra, but its corresponding equation of state behaves more like $N_{f}=2+1$ quark matter. These are consistent with the dimension analysis at ultraviolet (UV) boundary. Our results suggest that the dilaton field taking the simple quadratic form can be regarded as a candidate of dual theory for pure gluodynamics. Even though it is still difficult to find the dual theory of full QCD, the possible existence of dual theory of pure gluodynamics would be quite encouraging. 

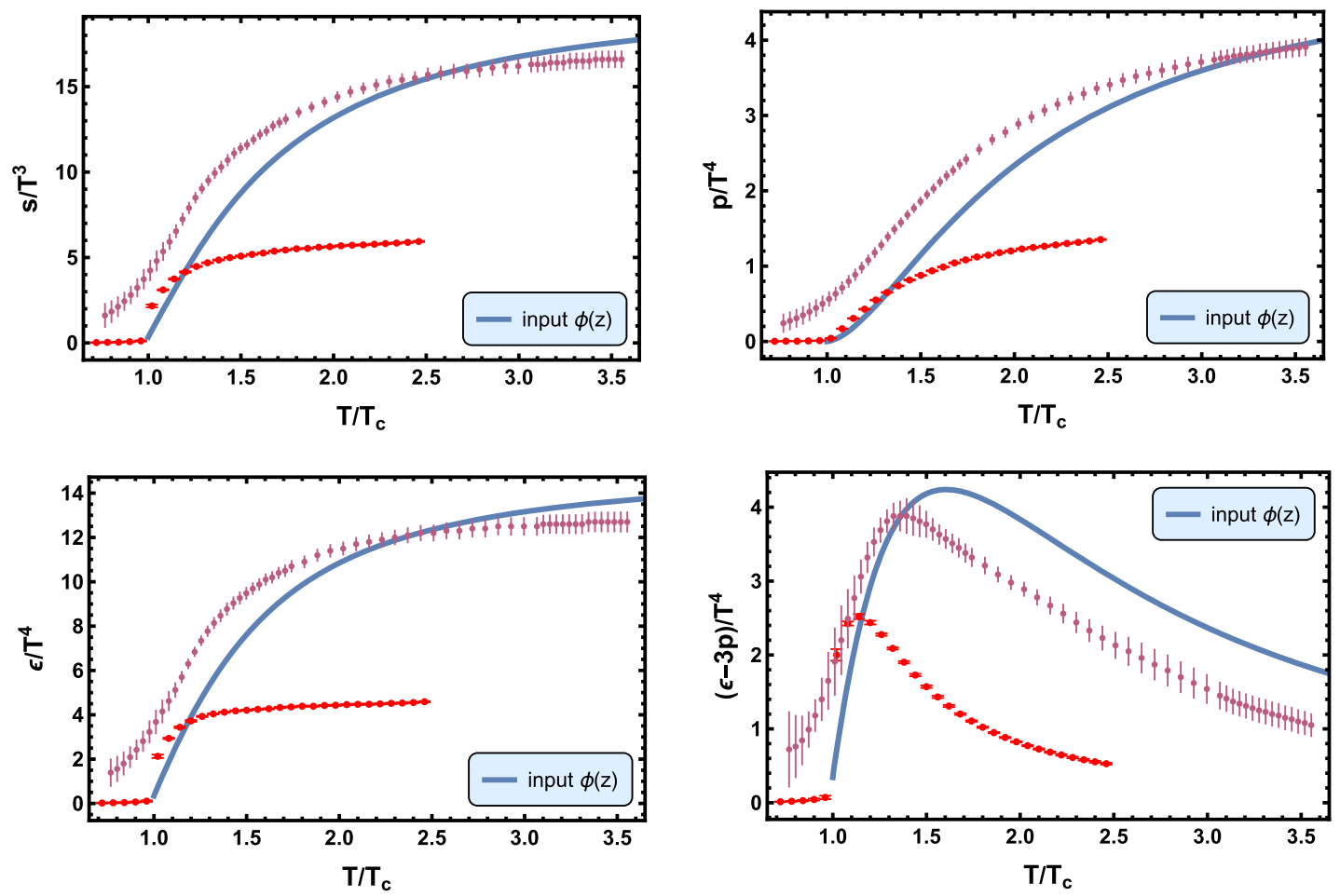

FIG. 8. The results of equation of state from model V. Upper left panel: the ratio of entropy density over cubic temperature as function of scaled temperature $T / T_{c}$. Upper right panel: the ratio of pressure over quartic temperature as function of scaled temperature $T / T_{c}$. Lower left panel: the energy density over quartic temperature as function of scaled temperature $T / T_{c}$. Lower right panel: the trace anomaly over quartic temperature as function of scaled temperature $T / T_{c}$. The blue line is for model $\mathrm{V}$, in which we input $\phi(z)$. The blue line is theoretical result from model $\mathrm{V}$, in which we input $\phi(z)$ and the parameters are $d=0.2463 \mathrm{GeV}^{2}$, and $G_{5}=0.39$. The red points are $S U(3)$ lattice data taken from Ref. [150] for pure gluon system, and the purple points are $N_{f}=2+1$ lattice data taken from Ref. [151].

\section{ACKNOWLEDGMENTS}

We thank Danning Li and Cong-Feng Qiao for helpful discussions. This work is supported in part by the National Natural Science Foundation of China (NSFC) Grants
No. 11735007, No. 11725523, and Chinese Academy of Sciences under Grant No. XDPB09, the start-up funding from University of Chinese Academy of Sciences(UCAS), and the Fundamental Research Funds for the Central Universities.
[1] M. Gell-Mann, Quarks, Acta Phys. Aust. Suppl. 9, 733 (1972).

[2] C. J. Morningstar and M. J. Peardon, The Glueball spectrum from an anisotropic lattice study, Phys. Rev. D 60, 034509 (1999).

[3] B. Lucini and M. Teper, SU(N) gauge theories in fourdimensions: Exploring the approach to $\mathrm{N}=$ infinity, J. High Energy Phys. 06 (2001) 050.

[4] H. B. Meyer, Glueball Regge trajectories, Ph.D. thesis, University of Oxford, Lincoln College (2004).

[5] Y. Chen et al., Glueball spectrum and matrix elements on anisotropic lattices, Phys. Rev. D 73, 014516 (2006).

[6] E. Gregory, A. Irving, B. Lucini, C. McNeile, A. Rago, C. Richards, and E. Rinaldi, Towards the glueball spectrum from unquenched lattice QCD, J. High Energy Phys. 10 (2012) 170.

[7] E. Bennett, D. K. Hong, J.-W. Lee, C. J. D. Lin, B. Lucini, M. Piai, and D. Vadacchino, $\mathrm{Sp}(4)$ gauge theory on the lattice: Towards SU(4)/Sp(4) composite Higgs (and beyond), J. High Energy Phys. 03 (2018) 185.

[8] E. Bennett, J. Holligan, D. K. Hong, J.-W. Lee, C. J. D. Lin, B. Lucini, M. Piai, and D. Vadacchino, Color dependence of tensor and scalar glueball masses in Yang-Mills theories, Phys. Rev. D 102, 011501 (2020).

[9] A. Athenodorou and M. Teper, The glueball spectrum of SU(3) gauge theory in $3+1$ dimensions, J. High Energy Phys. 11 (2020) 172. 
[10] E. Bennett, J. Holligan, D. K. Hong, J.-W. Lee, C. J. D. Lin, B. Lucini, M. Piai, and D. Vadacchino, Glueballs and strings in $S p(2 N)$ Yang-Mills theories, Phys. Rev. D 103, 054509 (2021).

[11] A. Athenodorou and M. Teper, SU(N) gauge theories in $3+1$ dimensions: glueball spectrum, string tensions and topology, J. High Energy Phys. 12 (2021) 082.

[12] N. Isgur and J. E. Paton, A flux tube model for hadrons in QCD, Phys. Rev. D 31, 2910 (1985).

[13] R. L. Jaffe and K. Johnson, Unconventional states of confined quarks and gluons, Phys. Lett. B 60, 201 (1976).

[14] T. Barnes, F. E. Close, and S. Monaghan, Hyperfine splittings of bag model gluonia, Nucl. Phys. B198, 380 (1982).

[15] T. Barnes, F. E. Close, and S. Monaghan, The MIT bag can accomodate the $\theta(1640)$ and iota (1440) as glueballs, Phys. Lett. 110B, 159 (1982).

[16] C. E. Carlson, T. H. Hansson, and C. Peterson, Meson, baryon and glueball masses in the MIT bag model, Phys. Rev. D 27, 1556 (1983).

[17] M. S. Chanowitz and S. R. Sharpe, Hybrids: Mixed states of quarks and gluons, Nucl. Phys. B222, 211 (1983); B228, 588(E) (1983).

[18] C. A. Dominguez and N. Paver, Local duality constraints on scalar gluonium, Z. Phys. C 31, 591 (1986).

[19] C. A. Dominguez and N. Paver, Tensor gluonium spectrum in QCD, Z. Phys. C 32, 391 (1986).

[20] J. I. Latorre, S. Narison, and S. Paban, $0++$ trigluonium sum rules, Phys. Lett. B 191, 437 (1987).

[21] S. Narison and G. Veneziano, QCD tests of $G$ (1.6) = Glueball, Int. J. Mod. Phys. A 04, 2751 (1989).

[22] S. Narison, Masses, decays and mixings of gluonia in QCD, Nucl. Phys. B509, 312 (1998).

[23] S. Narison, Masses, decays and mixings of gluonia in QCD, Nucl. Phys. B, Proc. Suppl. 64, 210 (1998).

[24] T. Huang, H.-Y. Jin, and A.-L. Zhang, Determination of the scalar glueball mass in QCD sum rules, Phys. Rev. D 59, 034026 (1999).

[25] S. Narison, Light scalar mesons in QCD, Nucl. Phys. B, Proc. Suppl. 186, 306 (2009).

[26] C.-F. Qiao and L. Tang, Finding the $0^{--}$Glueball, Phys. Rev. Lett. 113, 221601 (2014).

[27] L. Tang and C.-F. Qiao, Mass spectra of $0^{+-}, 1^{-+}$, and $2^{+-}$ exotic glueballs, Nucl. Phys. B904, 282 (2016).

[28] A. Pimikov, H.-J. Lee, N. Kochelev, and P. Zhang, Is the exotic $0^{--}$glueball a pure gluon state?, Phys. Rev. D 95, 071501 (2017).

[29] A. Pimikov, H.-J. Lee, and N. Kochelev, Comment on "Finding the $0^{--}$Glueball", Phys. Rev. Lett. 119, 079101 (2017).

[30] C.-F. Qiao and L. Tang, Reply to "Comment on 'Finding the $0^{--}$Glueball"' and comment on 'Is the exotic $0^{--}$ glueball a pure gluon state?', arXiv:1704.08589.

[31] A. Pimikov, H.-J. Lee, N. Kochelev, P. Zhang, and V. Khandramai, Exotic glueball $0^{ \pm-}$states in QCD sum rules, Phys. Rev. D 96, 114024 (2017).

[32] H.-X. Chen, W. Chen, and S.-L. Zhu, Toward the existence of the odderon as a three-gluon bound state, Phys. Rev. D 103, L091503 (2021).
[33] A. Szczepaniak, E. S. Swanson, C.-R. Ji, and S. R. Cotanch, Glueball Spectroscopy in a Relativistic Many Body Approach to Hadron Structure, Phys. Rev. Lett. 76, 2011 (1996).

[34] F. J. Llanes-Estrada, S. R. Cotanch, P. J. de A. Bicudo, J. E. F. T. Ribeiro, and A. P. Szczepaniak, QCD glueball Regge trajectories and the Pomeron, Nucl. Phys. A710, 45 (2002).

[35] F. J. Llanes-Estrada, P. Bicudo, and S. R. Cotanch, Oddballs and a Low Odderon Intercept, Phys. Rev. Lett. 96, 081601 (2006).

[36] D. V. Bugg, M. J. Peardon, and B. S. Zou, The Glueball spectrum, Phys. Lett. B 486, 49 (2000).

[37] Q. Zhao, B.-S. Zou, and Z.-B. Ma, Glueball-Q anti-Q mixing and Okuba-Zweig-Iizuka rule violation in the hadronic decays of heavy quarkonia, Phys. Lett. B 631, 22 (2005).

[38] H.-Y. Cheng, C.-K. Chua, and K.-F. Liu, Scalar glueball, scalar quarkonia, and their mixing, Phys. Rev. D 74, 094005 (2006).

[39] B. A. Li, Chiral field theory of 0-+ glueball, Phys. Rev. D 81, 114002 (2010).

[40] S. He, M. Huang, and Q.-S. Yan, The Pseudoscalar glueball in a chiral Lagrangian model with instanton effect, Phys. Rev. D 81, 014003 (2010).

[41] H.-Y. Cheng, Scalar and pseudoscalar glueballs revisited, AIP Conf. Proc. 1257, 477 (2010).

[42] S. Janowski, F. Giacosa, and D. H. Rischke, Is f0(1710) a glueball?, Phys. Rev. D 90, 114005 (2014).

[43] W. I. Eshraim, Phenomenology of a pseudoscalar glueball and charmed mesons, Ph.D. thesis, Frankfurt University, 2015.

[44] A. V. Sarantsev, I. Denisenko, U. Thoma, and E. Klempt, Scalar isoscalar mesons and the scalar glueball from radiative $J / \psi$ decays, Phys. Lett. B 816, 136227 (2021).

[45] V. Mathieu, N. Kochelev, and V. Vento, The physics of glueballs, Int. J. Mod. Phys. E 18, 1 (2009).

[46] E. Klempt and A. Zaitsev, Glueballs, hybrids, multiquarks. Experimental facts versus QCD inspired concepts, Phys. Rep. 454, 1 (2007).

[47] C. Amsler and N. A. Tornqvist, Mesons beyond the naive quark model, Phys. Rep. 389, 61 (2004).

[48] M. A. Braun, Odderon and QCD (1998).

[49] L. Lukaszuk and B. Nicolescu, A possible interpretation of pp rising total cross-sections, Lett. Nuovo Cimento 8, 405 (1973).

[50] V. M. Abazov et al., Odderon Exchange from Elastic Scattering Differences between $p p$ and $p \bar{p}$ Data at $1.96 \mathrm{TeV}$ and from pp Forward Scattering Measurements, Phys. Rev. Lett. 127, 062003 (2021).

[51] I. Szanyi, L. Jenkovszky, R. Schicker, and V. Svintozelskyi, Pomeron/glueball and odderon/oddball trajectories, Nucl. Phys. A998, 121728 (2020).

[52] T. Csörgő, T. Novak, R. Pasechnik, A. Ster, and I. Szanyi, Evidence of Odderon-exchange from scaling properties of elastic scattering at TeV energies, Eur. Phys. J. C 81, 180 (2021).

[53] T. Csörgó and I. Szanyi, Observation of Odderon effects at LHC energies: a real extended Bialas-Bzdak model study, Eur. Phys. J. C 81, 611 (2021). 
[54] I. Szanyi, N. Bence, and L. Jenkovszky, New physics from TOTEM's recent measurements of elastic and total cross sections, J. Phys. G 46, 055002 (2019).

[55] J. M. Maldacena, The large N limit of superconformal field theories and supergravity, Adv. Theor. Math. Phys. 2, 231 (1998).

[56] S. S. Gubser, I. R. Klebanov, and A. M. Polyakov, Gauge theory correlators from noncritical string theory, Phys. Lett. B 428, 105 (1998).

[57] E. Witten, Anti-de Sitter space and holography, Adv. Theor. Math. Phys. 2, 253 (1998).

[58] O. Aharony, S. S. Gubser, J. M. Maldacena, H. Ooguri, and Y. Oz, Large $\mathrm{N}$ field theories, string theory and gravity, Phys. Rep. 323, 183 (2000).

[59] O. Aharony, The non-AdS/non-CFT correspondence, or three different paths to QCD, in Progress in String, Field and Particle Theory (Springer Science+Business Media, Dordrecht, 2003).

[60] A. Zaffaroni, RTN lectures on the non AdS/non CFT correspondence, Proc. Sci., RTN2005 (2005) 005.

[61] J. Erdmenger, N. Evans, I. Kirsch, and E. Threlfall, Mesons in gauge/gravity duals-a review, Eur. Phys. J. A 35, 81 (2008).

[62] P. Kovtun, D. T. Son, and A. O. Starinets, Viscosity in Strongly Interacting Quantum Field Theories from Black Hole Physics, Phys. Rev. Lett. 94, 111601 (2005).

[63] U. Gursoy, E. Kiritsis, L. Mazzanti, and F. Nitti, Improved holographic Yang-Mills at finite temperature: Comparison with data, Nucl. Phys. B820, 148 (2009).

[64] T. Alho, M. Järvinen, K. Kajantie, E. Kiritsis, C. Rosen, and K. Tuominen, A holographic model for QCD in the Veneziano limit at finite temperature and density, J. High Energy Phys. 04 (2014) 124; 02 (2015) 033(E).

[65] A. Ballon-Bayona, L. A. H. Mamani, A. S. Miranda, and V. T. Zanchin, Effective holographic models for QCD: Thermodynamics and viscosity coefficients, Phys. Rev. D 104, 046013 (2021).

[66] M. Jarvinen and E. Kiritsis, Holographic models for QCD in the Veneziano limit, J. High Energy Phys. 03 (2012) 002.

[67] D. Li, M. Huang, and Q.-S. Yan, A dynamical soft-wall holographic QCD model for chiral symmetry breaking and linear confinement, Eur. Phys. J. C 73, 2615 (2013).

[68] T. Alho, M. Järvinen, K. Kajantie, E. Kiritsis, and K. Tuominen, On finite-temperature holographic QCD in the Veneziano limit, J. High Energy Phys. 01 (2013) 093.

[69] D. Li, J. Liao, and M. Huang, Enhancement of jet quenching around phase transition: result from the dynamical holographic model, Phys. Rev. D 89, 126006 (2014).

[70] D. Li, S. He, and M. Huang, Temperature dependent transport coefficients in a dynamical holographic QCD model, J. High Energy Phys. 06 (2015) 046.

[71] K. Chelabi, Z. Fang, M. Huang, D. Li, and Y.-L. Wu, Realization of chiral symmetry breaking and restoration in holographic QCD, Phys. Rev. D 93, 101901 (2016).

[72] Z. Fang, Y.-L. Wu, and L. Zhang, Chiral phase transition and meson spectrum in improved soft-wall AdS/QCD, Phys. Lett. B 762, 86 (2016).
[73] J. Erlich, E. Katz, D. T. Son, and M. A. Stephanov, QCD and a Holographic Model of Hadrons, Phys. Rev. Lett. 95, 261602 (2005).

[74] A. Karch, E. Katz, D. T. Son, and M. A. Stephanov, Linear confinement and AdS/QCD, Phys. Rev. D 74, 015005 (2006).

[75] T. Sakai and S. Sugimoto, Low energy hadron physics in holographic QCD, Prog. Theor. Phys. 113, 843 (2005).

[76] T. Sakai and S. Sugimoto, More on a holographic dual of QCD, Prog. Theor. Phys. 114, 1083 (2005).

[77] G. F. de Teramond and S. J. Brodsky, Hadronic Spectrum of a Holographic Dual of QCD, Phys. Rev. Lett. 94, 201601 (2005).

[78] L. Da Rold and A. Pomarol, Chiral symmetry breaking from five dimensional spaces, Nucl. Phys. B721, 79 (2005).

[79] K. Ghoroku, N. Maru, M. Tachibana, and M. Yahiro, Holographic model for hadrons in deformed $\operatorname{AdS}(5)$ background, Phys. Lett. B 633, 602 (2006).

[80] O. Andreev and V. I. Zakharov, Gluon condensate, Wilson loops and gauge/string duality, Phys. Rev. D 76, 047705 (2007).

[81] O. Andreev and V. I. Zakharov, Heavy-quark potentials and AdS/QCD, Phys. Rev. D 74, 025023 (2006).

[82] M. Kruczenski, L. A. Pando Zayas, J. Sonnenschein, and D. Vaman, Regge trajectories for mesons in the holographic dual of large-N(c) QCD, J. High Energy Phys. 06 (2005) 046.

[83] S. Kuperstein and J. Sonnenschein, Non-critical, near extremal AdS(6) background as a holographic laboratory of four dimensional YM theory, J. High Energy Phys. 11 (2004) 026.

[84] H. Forkel, M. Beyer, and T. Frederico, Linear square-mass trajectories of radially and orbitally excited hadrons in holographic QCD, J. High Energy Phys. 07 (2007) 077.

[85] D. K. Hong, T. Inami, and H.-U. Yee, Baryons in AdS/ QCD, Phys. Lett. B 646, 165 (2007).

[86] K. Nawa, H. Suganuma, and T. Kojo, Baryons in holographic QCD, Phys. Rev. D 75, 086003 (2007).

[87] D. K. Hong, M. Rho, H.-U. Yee, and P. Yi, Chiral dynamics of baryons from string theory, Phys. Rev. D 76, 061901 (2007).

[88] C. Csaki, H. Ooguri, Y. Oz, and J. Terning, Glueball mass spectrum from supergravity, J. High Energy Phys. 01 (1999) 017.

[89] R. de Mello Koch, A. Jevicki, M. Mihailescu, and J. P. Nunes, Evaluation of glueball masses from supergravity, Phys. Rev. D 58, 105009 (1998).

[90] M. Zyskin, A note on the glueball mass spectrum, Phys. Lett. B 439, 373 (1998).

[91] J. A. Minahan, Glueball mass spectra and other issues for supergravity duals of QCD models, J. High Energy Phys. 01 (1999) 020.

[92] C. Csaki, Y. Oz, J. Russo, and J. Terning, Large N QCD from rotating branes, Phys. Rev. D 59, 065012 (1999).

[93] C. Csaki and J. Terning, Glueball mass spectrum from supergravity, AIP Conf. Proc. 494, 321 (1999).

[94] R. C. Brower, S. D. Mathur, and C.-I. Tan, Glueball spectrum for QCD from AdS supergravity duality, Nucl. Phys. B587, 249 (2000). 
[95] H. Boschi-Filho and N. R. F. Braga, Gauge/string duality and scalar glueball mass ratios, J. High Energy Phys. 05 (2003) 009.

[96] H. Boschi-Filho and N. R. F. Braga, QCD/string holographic mapping and glueball mass spectrum, Eur. Phys. J. C 32, 529 (2004).

[97] R. Apreda, D. E. Crooks, N. J. Evans, and M. Petrini, Confinement, glueballs and strings from deformed AdS, J. High Energy Phys. 05 (2004) 065.

[98] H. Boschi-Filho, N. R. F. Braga, and H. L. Carrion, Glueball Regge trajectories from gauge/string duality and the Pomeron, Phys. Rev. D 73, 047901 (2006).

[99] P. Colangelo, F. De Fazio, F. Jugeau, and S. Nicotri, On the light glueball spectrum in a holographic description of QCD, Phys. Lett. B 652, 73 (2007).

[100] H. Forkel, Holographic glueball structure, Phys. Rev. D 78, 025001 (2008).

[101] E. F. Capossoli and H. Boschi-Filho, Odd spin glueball masses and the Odderon Regge trajectories from the holographic hardwall model, Phys. Rev. D 88, 026010 (2013).

[102] D. Li and M. Huang, Dynamical holographic QCD model for glueball and light meson spectra, J. High Energy Phys. 11 (2013) 088.

[103] L. Bellantuono, P. Colangelo, and F. Giannuzzi, Holographic oddballs, J. High Energy Phys. 10 (2015) 137.

[104] E. F. Capossoli and H. Boschi-Filho, Glueball spectra and Regge trajectories from a modified holographic softwall model, Phys. Lett. B 753, 419 (2016).

[105] E. F. Capossoli, D. Li, and H. Boschi-Filho, Pomeron and odderon Regge trajectories from a dynamical holographic model, Phys. Lett. B 760, 101 (2016).

[106] E. F. Capossoli, D. Li, and H. Boschi-Filho, Dynamical corrections to the anomalous holographic soft-wall model: The pomeron and the odderon, Eur. Phys. J. C 76, 320 (2016).

[107] D. M. Rodrigues, Eduardo Folco Capossoli, and Henrique Boschi-Filho, Twist two operator approach for even spin glueball masses and pomeron Regge trajectory from the Hardwall model, Phys. Rev. D 95, 076011 (2017).

[108] D. M. Rodrigues, E. Folco Capossoli, and H. Boschi-Filho, Scalar and higher even spin glueball masses from an anomalous modified holographic model, Europhys. Lett. 122, 21001 (2018).

[109] A. Ballon-Bayona, H. Boschi-Filho, L. A. H. Mamani, A. S. Miranda, and V.T. Zanchin, Effective holographic models for QCD: Glueball spectrum and trace anomaly, Phys. Rev. D 97, 046001 (2018).

[110] E. F. Capossoli, M. A. M. Contreras, D. Li, A. Vega, and H. Boschi-Filho, Hadronic spectra from deformed AdS backgrounds, Chin. Phys. C 44, 064104 (2020).

[111] M. Rinaldi and V. Vento, Meson and glueball spectroscopy within the graviton soft wall model, Phys. Rev. D 104, 034016 (2021).

[112] D. Elander, Glueball spectra of SQCD-like theories, J. High Energy Phys. 03 (2010) 114.

[113] D. Elander and M. Piai, Light scalars from a compact fifth dimension, J. High Energy Phys. 01 (2011) 026.
[114] M. Bianchi, M. Prisco, and W. Mueck, New results on holographic three point functions, J. High Energy Phys. 11 (2003) 052.

[115] M. Berg, M. Haack, and W. Mueck, Bulk dynamics in confining gauge theories, Nucl. Phys. B736, 82 (2006).

[116] M. Berg, M. Haack, and W. Mueck, Glueballs vs. gluinoballs: Fluctuation spectra in nonAdS/Non-CFT, Nucl. Phys. B789, 1 (2008).

[117] D. Elander and M. Piai, On the glueball spectrum of walking backgrounds from wrapped-D5 gravity duals, Nucl. Phys. B871, 164 (2013).

[118] D. Elander and M. Piai, Calculable mass hierarchies and a light dilaton from gravity duals, Phys. Lett. B 772, 110 (2017).

[119] D. Elander and M. Piai, Glueballs on the Baryonic Branch of Klebanov-Strassler: Dimensional deconstruction and a light scalar particle, J. High Energy Phys. 06 (2017) 003.

[120] D. Elander, A. F. Faedo, D. Mateos, D. Pravos, and J. G. Subils, Mass spectrum of gapped, non-confining theories with multi-scale dynamics, J. High Energy Phys. 05 (2019) 175.

[121] D. Elander, M. Piai, and J. Roughley, Holographic glueballs from the circle reduction of Romans supergravity, J. High Energy Phys. 02 (2019) 101.

[122] D. Elander, M. Piai, and J. Roughley, Probing the holographic dilaton, J. High Energy Phys. 06 (2020) 177; 12 (2020) 109(E).

[123] D. Elander, M. Piai, and J. Roughley, Dilatonic states near holographic phase transitions, Phys. Rev. D 103, 106018 (2021).

[124] D. Elander, M. Frigerio, M. Knecht, and J.-L. Kneur, Holographic models of composite Higgs in the Veneziano limit. Part I. Bosonic sector, J. High Energy Phys. 03 (2021) 182.

[125] D. Elander, M. Piai, and J. Roughley, Light dilaton in a metastable vacuum, Phys. Rev. D 103, 046009 (2021).

[126] D. Elander, M. Piai, and J. Roughley, Coulomb branch of $N=4$ SYM and dilatonic scions in supergravity, Phys. Rev. D 104, 046003 (2021).

[127] K. Hashimoto, C.-I. Tan, and S. Terashima, Glueball decay in holographic QCD, Phys. Rev. D 77, 086001 (2008).

[128] F. Brünner, D. Parganlija, and A. Rebhan, Glueball decay rates in the Witten-Sakai-Sugimoto model, Phys. Rev. D 91, 106002 (2015); 93, 109903(E) (2016).

[129] F. Brünner and A. Rebhan, Nonchiral Enhancement of Scalar Glueball Decay in the Witten-Sakai-Sugimoto Model, Phys. Rev. Lett. 115, 131601 (2015).

[130] M. Huang and D. Li, Dynamical holographic QCD model: Resembling renormalization group from ultraviolet to infrared, Springer Proc. Phys. 170, 367 (2016).

[131] S. S. Gubser and A. Nellore, Mimicking the QCD equation of state with a dual black hole, Phys. Rev. D 78, 086007 (2008).

[132] S. S. Gubser, A. Nellore, S. S. Pufu, and F. D. Rocha, Thermodynamics and Bulk Viscosity of Approximate Black Hole Duals to Finite Temperature Quantum Chromodynamics, Phys. Rev. Lett. 101, 131601 (2008).

[133] O. DeWolfe, S. S. Gubser, and C. Rosen, A holographic critical point, Phys. Rev. D 83, 086005 (2011). 
[134] U. Gursoy and E. Kiritsis, Exploring improved holographic theories for QCD: Part I, J. High Energy Phys. 02 (2008) 032.

[135] U. Gursoy, E. Kiritsis, and F. Nitti, Exploring improved holographic theories for QCD: Part II, J. High Energy Phys. 02 (2008) 019.

[136] U. Gursoy, E. Kiritsis, L. Mazzanti, G. Michalogiorgakis, and F. Nitti, Improved holographic QCD, Lect. Notes Phys. 828, 79 (2011).

[137] Y. Yang and P.-H. Yuan, A refined holographic QCD model and QCD phase structure, J. High Energy Phys. 11 (2014) 149.

[138] D. Dudal and S. Mahapatra, Thermal entropy of a quarkantiquark pair above and below deconfinement from a dynamical holographic QCD model, Phys. Rev. D 96, 126010 (2017).

[139] D. Li, S. He, M. Huang, and Q.-S. Yan, Thermodynamics of deformed $\mathrm{AdS}_{5}$ model with a positive/negative quadratic correction in graviton-dilaton system, J. High Energy Phys. 09 (2011) 041.

[140] H. Weyl and H. L. Brose, Raum-zeit-materie [spacetime-matter], Lectures on General Relativity (in German) (Springer, Berlin, 1921).

[141] H. Weyl and J. Ehlers, Space, Time, Matter: Lectures on General Relativity (Springer, Berlin, Germany, 1993).

[142] U. Gursoy, E. Kiritsis, L. Mazzanti, and F. Nitti, Holography and thermodynamics of 5D dilaton-gravity, J. High Energy Phys. 05 (2009) 033.

[143] Actually, the black hole may not exist for all values of temperature and chemical potential. Under some situations, it only exists above a certain temperature $T_{\min }$. The AdS thermal gas is the only solution below $T_{\min }$. However, in other situations, we always have the black hole solution.
[144] E. F. Capossoli, D. Li, and H. Boschi-Filho, Pomeron and odderon regge trajectories from a dynamical holographic model, Phys. Lett. B 760, 101 (2016).

[145] A. Amorim, R. Carcassés Quevedo, and M. S. Costa, Nonminimal coupling contribution to DIS at low $x$ in holographic QCD, Phys. Rev. D 98, 026016 (2018).

[146] Y. Chen and M. Huang, Two-gluon and trigluon glueballs from dynamical holography QCD, Chin. Phys. C 40, 123101 (2016).

[147] P. H. Richter, Estimating errors in least-squares fitting, Telecommun. Data Acquisition Prog. Rep. 42, 107 (1995).

[148] Please remember Eq. (8). This value of $b$ means $\Phi(z)=\left(1 \mathrm{GeV}^{2}\right) z^{2}$.

[149] R. Critelli, J. Noronha, J. Noronha-Hostler, I. Portillo, C. Ratti, and R. Rougemont, Critical point in the phase diagram of primordial quark-gluon matter from black hole physics, Phys. Rev. D 96, 096026 (2017).

[150] M. Caselle, A. Nada, and M. Panero, QCD thermodynamics from lattice calculations with nonequilibrium methods: The SU(3) equation of state, Phys. Rev. D 98, 054513 (2018).

[151] S. Borsanyi, Z. Fodor, C. Hoelbling, S. D. Katz, S. Krieg, and K. K. Szabo, Full result for the QCD equation of state with $2+1$ flavors, Phys. Lett. B 730, 99 (2014).

[152] As explained in Ref. [149], the characteristic energy scale $\Lambda$, the energy dimension of which is 1 , is introduced to express dimensionful observables in the physical unit. When a gauge/gravity observable with dimension $[E]^{q}$ is expressed in the physical unit, it should be multiplied by $\Lambda^{q}$.

[153] J. Grefa, J. Noronha, J. Noronha-Hostler, I. Portillo, C. Ratti, and R. Rougemont, Hot and dense quark-gluon plasma thermodynamics from holographic black holes, Phys. Rev. D 104, 034002 (2021). 